OPEN ACCESS

Edited by:

Loïc Riviere,

Université de Bordeaux, France

Reviewed by:

Chiranjib Pal,

West Bengal State University, India

Laurence A. Marchat,

Instituto Politécnico Nacional, Mexico

*Correspondence:

Ying Zhang

yzhang207@zju.edu.cn

Specialty section:

This article was submitted to

Parasite and Host.

a section of the journal

Frontiers in Cellular

and Infection Microbiology

Received: 01 November 2020

Accepted: 08 January 2021

Published: 08 March 2021

Citation:

Zhang Y, Alvarez-Manzo H, Leone J,

Schweig $S$ and Zhang $Y$ (2021)

Botanical Medicines Cryptolepis

sanguinolenta, Artemisia annua,

Scutellaria baicalensis, Polygonum

cuspidatum, and Alchornea cordifolia

Demonstrate Inhibitory Activity

Against Babesia duncani.

Front. Cell. Infect. Microbiol. 11:624745.

doi: 10.3389/fcimb.2021.624745

\section{Botanical Medicines Cryptolepis sanguinolenta, Artemisia annua, Scutellaria baicalensis, Polygonum cuspidatum, and Alchornea cordifolia Demonstrate Inhibitory Activity Against Babesia duncani}

\author{
Yumin Zhang ${ }^{1}$, Hector Alvarez-Manzo ${ }^{1}$, Jacob Leone ${ }^{2}$, Sunjya Schweig ${ }^{3}$ \\ and Ying Zhang ${ }^{4 *}$

\begin{abstract}
Department of Molecular Microbiology and Immunology, Johns Hopkins Bloomberg School of Public Health, Johns Hopkins University, Baltimore, MD, United States, ${ }^{2}$ FOCUS Health Group, Naturopathic, Novato, CA, United States, ${ }^{3}$ California Center for Functional Medicine, Kensington, CA, United States, ${ }^{4}$ State Key Laboratory for the Diagnosis and Treatment of Infectious Diseases, National Clinical Research Center for Infectious Diseases, The First Affiliated Hospital, Zhejiang University School of Medicine, Hangzhou, China
\end{abstract}

Human babesiosis is a CDC reportable disease in the United States and is recognized as an emerging health risk in multiple parts of the world. The current treatment for human babesiosis is suboptimal due to treatment failures and unwanted side effects. Although Babesia duncani was first described almost 30 years ago, further research is needed to elucidate its pathogenesis and clarify optimal treatment regimens. Here, we screened a panel of herbal medicines and identified Cryptolepis sanguinolenta, Artemisia annua, Scutellaria baicalensis, Alchornea cordifolia, and Polygonum cuspidatum to have good in vitro inhibitory activity against $B$. duncani in the hamster erythrocyte model. Furthermore, we found their potential bioactive compounds, cryptolepine, artemisinin, artesunate, artemether, and baicalein, to have good activity against $B$. duncani, with $\mathrm{IC}_{50}$ values of $3.4 \mu \mathrm{M}, 14 \mu \mathrm{M}, 7.4 \mu \mathrm{M}, 7.8 \mu \mathrm{M}$, and $12 \mu \mathrm{M}$, respectively, which are comparable or lower than that of the currently used drugs quinine $(10 \mu \mathrm{M})$ and clindamycin $(37 \mu \mathrm{M})$. B. duncani treated with cryptolepine and quinine at their respective $1 \times, 2 \times, 4 \times$ and $8 \times 1 C_{50}$ values, and by artemether at $8 \times \mathrm{IC}_{50}$ for three days could not regrow in subculture. Additionally, Cryptolepis sanguinolenta 90\% ethanol extract also exhibited no regrowth after 6 days of subculture at doses of $2 \times, 4 \times$, and $8 \times 1 \mathrm{IC}_{50}$ values. Our results indicate that some botanical medicines and their active constituents have potent activity against $B$. duncani in vitro and may be further explored for more effective treatment of babesiosis.

Keywords: Babesia duncani, Cryptolepis sanguinolenta, herbal medicine, cryptolepine, artemisinin, baicalein, phytonutrient, phytotherapy 


\section{INTRODUCTION}

Babesiosis is a disease caused by the Babesia parasite that infects red blood cells. Babesia genus belongs to the apicomplexan phylum, and over 100 Babesia species have been identified (Birkenheuer et al., 2006). However, only a few of these have been documented to infect humans. Babesia divergens, Babesia microti, and Babesia duncani cause the most human babesiosis cases worldwide. Most human babesiosis infections in the United States are caused by B. microti and B. duncani, and in Europe the majority of reported cases are due to $B$. divergens (Vannier and Krause, 2012). All of these Babesia species can be acquired by Ixodes ticks that are also reservoirs of Borrelia spirochetes which cause Lyme disease. Recently, Dermacentor albipictus has been implicated as a competent tick vector for Babesia duncani (Swei et al., 2019). Epidemiologic studies have documented that up to $23 \%$ of patients with babesiosis also experienced concurrent Lyme disease (Diuk-Wasser et al., 2016) and coinfected patients can experience a greater number of symptoms for a longer duration than those with Lyme disease alone (Krause et al., 1996; Krause et al., 2002). In addition to transmission by tick bite, human babesiosis transmission has also been reported via blood transfusion, organ transplantation, and vertical transmission during pregnancy (Lux et al., 2003; Leiby, 2006). The clinical manifestations of babesiosis range from asymptomatic to severe and common clinical symptoms in patients include fever, chills, and sweats. Patients with immunological diseases, on immunosuppressive therapies, and those who have undergone splenectomy are at increased risk of more severe symptoms and even death (Vannier et al., 2015).

Babesiosis caused by Babesia microti is endemic in the Northeast and the upper Midwest of the United States. Babesiosis caused by B. duncani was first described in Washington State as strain WA1 in 1991 and is widespread in North America based on molecular and immunological tests (Vannier and Krause, 2012; Scott and Scott, 2018). Animals such as mice, gerbils, and hamsters are susceptible to infection with $B$. duncani via intraperitoneal injection. Studies in animal models show that B. duncani has a different pathogenesis than B. microti, in that the parasitemia in hamsters infected with B. microti can initially be very high, but then decrease to undetectable levels within several weeks. In contrast, $B$. duncani can cause severe acute disease and death in hamsters, within 10 days after inoculation (Kjemtrup and Conrad, 2000). In mouse models, $B$. duncani can give rise to more severe presentations and higher mortality rates than B. microti (Moro et al., 1998). In terms of morphology, there is no obvious difference in intraerythrocytic stages between B. duncani and B. microti, nevertheless, based on the phylogenetic analysis of 18S RNA gene, ITS2 gene, $c y t b$ gene, and coxI gene, B. duncani show a distinct evolutionary lineage compared to B. microti and other Babesia species (Virji et al., 2019).

The current treatment protocols for human babesiosis frequently use medications such as atovaquone, azithromycin, clindamycin, quinine, and their combinations (Smith et al., 2020). However, these regimens are suboptimal and are associated with treatment failures and significant side effects
(Kjemtrup and Conrad, 2000), even in immunocompetent patients (Krause et al., 1998; Gonzalez et al., 2014). Some cases of Babesia infection can be persistent, and recrudescence may occur up to two years after therapy (Krause et al., 2008; Raffalli and Wormser, 2016). Furthermore, it has been demonstrated that $B$. duncani showed unexpectedly high tolerance to atovaquone, azithromycin, clindamycin, and quinine in vitro (Abraham et al., 2018), and B. duncani could regrow in vitro after exposure to high concentrations of atovaquone or azithromycin for 3 days (Zhang et al., 2020). Therefore, alternative more effective antimicrobial agents and treatment regimens with fewer side effects need to be developed.

In the past decade, some promising anti-parasite drug candidates or compounds have been identified against $B$. microti, $B$. divergens, $B$. gibsoni, B. bovis, B. bigemina, and $B$. caballi in vitro or in vivo including mycophenolic acid, pentamidine, doxorubicin hydrochloride, vorinostat, luteolin, pyronaridine, robenidine, primaquine, and diphenyl furan for potential medical and veterinary treatment (Nehrbass-Stuedli et al., 2011; Rizk et al., 2015; Yao et al., 2015; Rizk et al., 2018; Li et al., 2020; Rizk et al., 2020). However, to date, limited research has focused on drug screening against $B$. duncani.

Herbal medicines were used by ancient cultures and their safety and efficacy have been documented by various traditional medicine systems such as Ayurveda and Traditional Chinese Medicine (Borchardt, 2002; Jaiswal et al., 2016). The adverse effects of botanical products were determined to be rare according to a recent report (Di Lorenzo et al., 2015). In a previous study, we have screened a panel of essential oils and found that garlic oil, black pepper, and their constituents showed good activity against $B$. duncani in a hamster erythrocyte model (Zhang et al., 2020). These findings indicated that natural products extracted from plants and herbs may serve as a potential source of promising compounds for anti-parasitic drugs. In this current study, we used the same hamster erythrocyte model to screen for inhibition of $B$. duncani using a panel of 46 herbal medicine extracts, many of which are useful in the treatment of babesiosis and related conditions according to the Buhner book on herbal medicines (Buhner, 2015) or were shown in our previous in vitro studies to have antimicrobial effects (Feng J. et al., 2020). Our results identified Cryptolepis sanguinolenta, Artemisia annua, Scutellaria baicalensis, Alchornea cordifolia, and Polygonum cuspidatum and their bioactive compounds as having good activity against B. duncani.

\section{MATERIALS AND METHODS}

\section{Hamster Donor Blood and Babesia duncani Culture}

Hamster whole blood was collected from Golden Syrian hamsters (Charles River) by cardiac puncture using phosphate buffer saline containing $15 \mathrm{mM}$ EDTA as anticoagulant according to protocols approved by the Johns Hopkins Institutional Animal Care and Use Committee. Hamster whole Blood was washed three times by centrifugation at $500 \mathrm{~g}$ for 
15 min in PBS, 15mM EDTA solution with careful removal of the supernatant and buffy coat at each wash. After the last wash, red blood cells (RBCs) were resuspended at a concentration of 50\% hematocrit in Puck's saline glucose buffer with extra glucose (20 g glucose $/ \mathrm{L})$ and stored at $4^{\circ} \mathrm{C}$ less than 2 weeks before use. Babesia duncani strain WA1 (ATCC PRA-302) was cultured in vitro in HaRGM medium with fresh hamster erythrocytes at $37^{\circ} \mathrm{C}$ under an atmosphere of $5 \% \mathrm{CO}_{2}$ with $95 \%$ humidity. Hamster erythrocytes were prepared with HaRGM medium, and continuous $B$. duncani culture method was performed as described in detail in our previous publication (Zhang et al., 2020).

\section{Natural Products and Compounds}

A panel of 46 herbal medicine extracts (Table 1) and relevant solvent controls were used for testing. The herbal medicine extracts of Uncaria tomentosa, Stevia rebaudiana, Juglans nigra, Cryptolepis sanguinolenta, Artemisia annua, Andrographis paniculata, Dipsacus fullonum, Withania somnifera, Scutellaria baicalensis, and Polygonum cuspidatum were sourced from KW Botanicals (San Anselmo, California) and Heron Botanicals (Kingston, Washington). Most of these natural products were provided as ethanol extracts at 30\%,60\%, and 90\% ethanol, and the ethanol solvent was also tested as a control in the respective concentrations. Other herbal medicine extracts were purchased commercially. Detailed information including source, extraction parts, extraction type, and manufacturer of all the herbal medicine extracts tested in this study is shown in Supplementary Table S1. Cryptolepine (cryptolepine hydrate; Sigma-Aldrich), artemisinin (Alfa Aesar), artesunate (TCI Chemicals), artemether (Frontier Scientific), baicalein (5,6,7-Trihydroxyflavone; Alfa Aesar), quinine (quinine hydrochloride; Frontier Scientific), and clindamycin (clindamycin hydrochloride; Sigma-Aldrich) were prepared in DMSO stock in high concentrations and then diluted to desired concentrations with DMSO.

\section{In Vitro Evaluation of Herbal Medicines and Compounds on Inhibition of $B$. duncani}

Babesia duncani was cultured in 24 -well plate at $2.5 \%$ hematocrit of hamster erythrocytes at $37^{\circ} \mathrm{C}$ under an atmosphere of $5 \% \mathrm{CO}_{2}$ with $95 \%$ humidity for 3-4 days. When parasitemia reached $2 \%$, the infected erythrocytes were split evenly into 96-well plates at $2.5 \%$ hematocrit each well for natural products and compounds test. Natural products were prepared in $10 \%(\mathrm{v} / \mathrm{v})$ DMSO stock solution. Ethanol at $30 \%$, $60 \%$, and $90 \%$ was prepared in parallel in DMSO as controls. Then the natural products and ethanol controls were added to 96-well plates containing infected erythrocytes to obtain final concentrations of $0.01 \%$. Treated cultures were inoculated at $37^{\circ} \mathrm{C}$ for 3 days without replacing medium in a chamber under an atmosphere of $5 \% \mathrm{CO}_{2}$ with $95 \%$ humidity. Then $1 \mu \mathrm{l}$ of erythrocytes laid on the plate bottom were taken out for growth test by SYBR Green I assay as described previously (Zhang et al., 2020). Briefly, the $1 \mu \mathrm{l}$ of RBCs sediment were placed into $100 \mu \mathrm{l}$ of lysis buffer consisting of $20 \mathrm{mM}$ Tris, $\mathrm{pH}$ 7.4, $5 \mathrm{mM}$
EDTA, $0.008 \%$ saponin, $0.08 \%$ Triton $\mathrm{X}-100$, and $2 \times$ SYBR Green I (10,000× stock, Invitrogen) in 96-well plates. The plates were then inoculated in the dark at $37^{\circ} \mathrm{C}$ for 60 mins followed by plate reading at excitation wavelength $(490 \mathrm{~nm})$ and a fluorescence intensity at $520 \mathrm{~nm}$ in a microplate reader (HTS 7000 plus Bio Assay Reader, PerkinElmer Inc., USA). For the $\mathrm{IC}_{50}$ susceptibility assay, $B$. duncani cultures were exposed to increasing concentrations of Artemisia annua (30\% ethanol extract), Cryptolepis sanguinolenta (90\% ethanol extract), Scutellaria baicalensis (90\% ethanol extract), cryptolepine, artemisinin, artesunate, artemether, baicalein, quinine, and clindamycin. Each $\mathrm{IC}_{50}$ was performed in triplicate in 96-well plates with a starting 2\% parasitemia and 2.5\% hematocrit followed by SYBR Green I stain. Dose-response curves with fitting a nonlinear regression curve were performed in GraphPad Prism (version 7.0) and then calculate the best-fit $\mathrm{IC}_{50}$ values. The morphology of Giemsa stained thin blood smears of $B$. duncani after treatment with compounds at $1 \times, 2 \times$, and $4 \times \mathrm{IC}_{50}$ concentrations for 3 days was observed under BZX710 All-in-One Fluorescence Microscope (KEYENCE, Inc., Itasca, IL, USA).

\section{Subculture Studies to Evaluate Killing Efficacy of the Top Natural Product Hits and Selected Compounds}

B. duncani cultures were exposed to Artemisia annua (30\% ethanol extract), Cryptolepis sanguinolenta (90\% ethanol extract), Scutellaria baicalensis (90\% ethanol extract), cryptolepine, artemisinin, artesunate, artemether, baicalein, quinine, and clindamycin at their respective $1 \times, 2 \times, 4 \times$ and $8 \times$ $\mathrm{IC}_{50}$ values for 3 days. After three days of exposure, the treated erythrocytes were washed three times in HaRGM medium to wash away drugs and then inoculated in fresh hamster erythrocytes at ratio of 1:5. Each compound was tested in triplicate, and every well had 4-6 replicates for sampling to avoid obvious hematocrit decrease. Culture conditions of the subcultures were identical to those described above. One microliter of erythrocytes laid on the plate bottom was taken out every one or two days and stored at $-80^{\circ} \mathrm{C}$. The growth of the subculture was examined by the SYBR Green I assay as described above, and the fluorescence units of day zero were normalized as zero.

\section{RESULTS}

\section{Evaluation of Natural Product Extracts for Inhibitory Activity Against B. duncani}

We evaluated a panel of 46 herbal medicine extracts and their corresponding controls at a concentration of $0.01 \%(\mathrm{v} / \mathrm{v})$ for inhibitory activity against $B$. duncani at an initial parasitemia of $2 \%$ in vitro after 3 days of incubation (Table 1). Cryptolepis sanguinolenta (60, 90\% ethanol extracts), Artemisia annua (30\% ethanol extract), Scutellaria baicalensis (60\%, 90\% ethanol extracts), Polygonum cuspidatum (60\% ethanol extract), and Alchornea cordifolia were the top hits with more than $50 \%$ 
TABLE 1 | Evaluation of a panel of 46 herbal medicines at $0.01 \%(v / v)$ for inhibitory activity against $B$. duncani after 3 days of incubation.

Product Names

Chinese Skullcap (90\% EE)

Cryptolepis (90\% EE)

Cryptolepis (60\% EE)

Chinese Skullcap (60\% EE)

Japanese knotweed (60\% EE)

Sweet wormwood (30\% EE)

Alchornea

Japanese knotweed (90\% EE)

Andrographis (90\% EE)

Andrographis (60\% EE)

Sweet wormwood (60\% EE)

Andrographis (30\% EE)

Cistus

Ashwagandha (30\% EE)

Hemp oil

Barberry

Chuan xin lian

Black walnut (90\% EE)

Ashwagandha (60\% EE)

Stevia/Tian ju ye

Ashwagandha (90\% EE)

Echinacea

Andrographis

Licorice

Grapefruit seed extract

Usnea

Eleuthero

Black walnut (60\% EE)

Sweet wormwood (90\% EE)

Gou teng

Teasel/Gao liang jiang

Osha

Cryptolepis (30\% EE)

Chinese Skullcap (30\% EE)

Houttuynia

Bidens

Ban zhi lian

Black walnut (30\% EE)

Uncaria

Samento

Cumanda

Banderol

Coptis

Japanese knotweed (30\% EE)

Reishi

Black Walnut
Plants

Inhibition (\%)

Scutellaria baicalensis

Cryptolepis sanguinolenta

Cryptolepis sanguinolenta

Scutellaria baicalensis

Polygonum cuspidatum

Artemisia annua

Alchornea cordifolia

Polygonum cuspidatum

Andrographis paniculata

Andrographis paniculata

Artemisia annua

Andrographis paniculata

Cistus incanus

Withania somnifera

Cannabis sativa

Berberis vulgaris

Andrographis paniculata

Juglans nigra

Withania somnifera

Stevia rebaudiana fol

Withania somnifera

Echinacea purpurea \& Echinacea angustifolia

Andrographis paniculata

Glycyrrhiza spp.

Grapefruit seed

Usnea spp.

Eleutherococcus senticosus

Juglans nigra

Artemisia annua

Uncaria rhynchophylla

Dipsacus fullonum

Ligusticum porter root

Cryptolepis sanguinolenta

Scutellaria baicalensis

Houttuynia

Bidens pilosa

Scutellaria barbata

Juglans nigra

Uncaria tomentosa

Uncaria tomentosa

Campsiandra angustifolia

Otoba sp.

Rhizoma coptidis

Polygonum cuspidatum

Ganoderma linghzi

Juglans nigra fruc

The growth of infected erythrocytes at day zero was set as 0\%. The growth of B. duncani in infected erythrocytes treated with $1 \%$ DMSO vehicle at day three was set as $100 \%$.

EE, ethanol extract. The bold product names indicate the effective hits studied in this study.

inhibitory effect at $0.01 \%$ against $B$. duncani. As a control, we tested the ethanol carrier at concentrations of $30 \%, 60 \%$, and $90 \%$, which did not show obvious inhibitory effect at up to $1 \%$ concentration.

Cryptolepis sanguinolenta, Artemisia annua, and Scutellaria baicalensis showed activity at 30\%,60\%, and 90\% EE, therefore, we further tested the half-maximal inhibitory concentration values of these three active natural product extracts at different ethanol concentrations. Dose-response assays confirmed that Cryptolepis sanguinolenta and Scutellaria baicalensis at $60 \%$ and $90 \%$ EE showed approximately 4-10 times lower $\mathrm{IC}_{50}$ values compared to those at $30 \% \mathrm{EE}$. In contrast, Artemisia annua at $30 \% \mathrm{EE}$ exhibited the lowest $\mathrm{IC}_{50}$ value, even lower than it was at $60 \%$ and $90 \%$ EE (Table 2; Figure 1).

\section{Artemisinin, Cryptolepine, and Baicalein Are the Active Constituents Extracted From Artemisia annua, Cryptolepis sanguinolenta, and Scutellaria baicalensis Respectively and Have High Potent Antibabesial Activity}

Since Cryptolepis sanguinolenta (90\% ethanol extract), Artemisia annua (30\% ethanol extract), and Scutellaria baicalensis (90\% 
TABLE 2 | The differing $I_{50}$ values indicated that Cryptolepis sanguinolenta Artemisia annua, and Scutellaria baicalensis showed different inhibitory effects in $30 \%, 60 \%$, and $90 \%$ ethanol extracts.

\begin{tabular}{lc} 
Natural product extracts & IC $_{\mathbf{5 0}}$ values \\
\hline Cryptolepis sanguinolenta (30\% EE) & $0.039 \%(\mathrm{v} / \mathrm{v})$ \\
Cryptolepis sanguinolenta (60\% EE) & $0.0041 \%$ \\
Cryptolepis sanguinolenta (90\% EE) & $0.0046 \%$ \\
Artemisia annua (30\% EE) & $0.0091 \%$ \\
Artemisia annua (60\% EE) & $0.0097 \%$ \\
Artemisia annua (90\% EE) & $0.030 \%$ \\
Scutellaria baicalensis (30\% EE) & $0.034 \%$ \\
Scutellaria baicalensis (60\% EE) & $0.038 \%$ \\
Scutellaria baicalensis (90\% EE) & $0.0097 \%$ \\
Cryptolepine & $3.4 \mu \mathrm{M}$ \\
Artemisinin & $14 \mu \mathrm{M}$ \\
Baicalein & $12 \mu \mathrm{M}$ \\
Quinine & $10 \mu \mathrm{M}$ \\
Clindamycin & $37 \mu \mathrm{M}$ \\
\hline
\end{tabular}

Growth was evaluated by SYBR Green stain at day three after B. duncani exposure to natural product extracts. Each natural product concentration was made in triplicate. $I C_{50}$ values were calculated in GraphPad Prism (version 7.0). EE, ethanol extract.

ethanol extract) extracts showed better inhibitory activity against B. duncani than Polygonum cuspidatum, and Alchornea cordifolia, we focused on testing cryptolepine, artemisinin, and baicalein, which are known bioactive compounds derived from these three herbal medicines (Klayman, 1993; Cimanga et al., 1996; Nishioka et al., 1998). For comparison, we also tested the commonly used clinical drugs quinine and clindamycin. All these compounds and drugs inhibited the growth of $B$. duncani in a dose-dependent manner (Figure 1). The $\mathrm{IC}_{50}$ values of cryptolepine, artemisinin, and baicalein were $3.4 \mu \mathrm{M}, 14 \mu \mathrm{M}$, and $12 \mu \mathrm{M}$, respectively. In contrast, the $\mathrm{IC}_{50}$ values of quinine and clindamycin were $10 \mu \mathrm{M}$ and $37 \mu \mathrm{M}$.

\section{Artemisinin Derivatives Artesunate and Artemether Showed Higher Inhibitory Activity Against B. duncani Than Artemisinin}

Artemisinin derivatives, including artesunate and artemether, are potent antimalarial drugs which have been shown to reduce malaria parasitemia more rapidly than any other known antimalarial drugs, and are effective against multidrug-resistant malaria parasites (Olliaro et al., 2001). In our research, we compared artemisinin, artesunate, and artemether for their inhibitory effects against $B$. duncani in our culture system (Figure 2). The $\mathrm{IC}_{50}$ values of artesunate and artemether were $7.4 \mu \mathrm{M}$ and $7.8 \mu \mathrm{M}$, respectively, indicating that both of the artemisinin derivatives showed higher inhibitory activity than artemisinin.
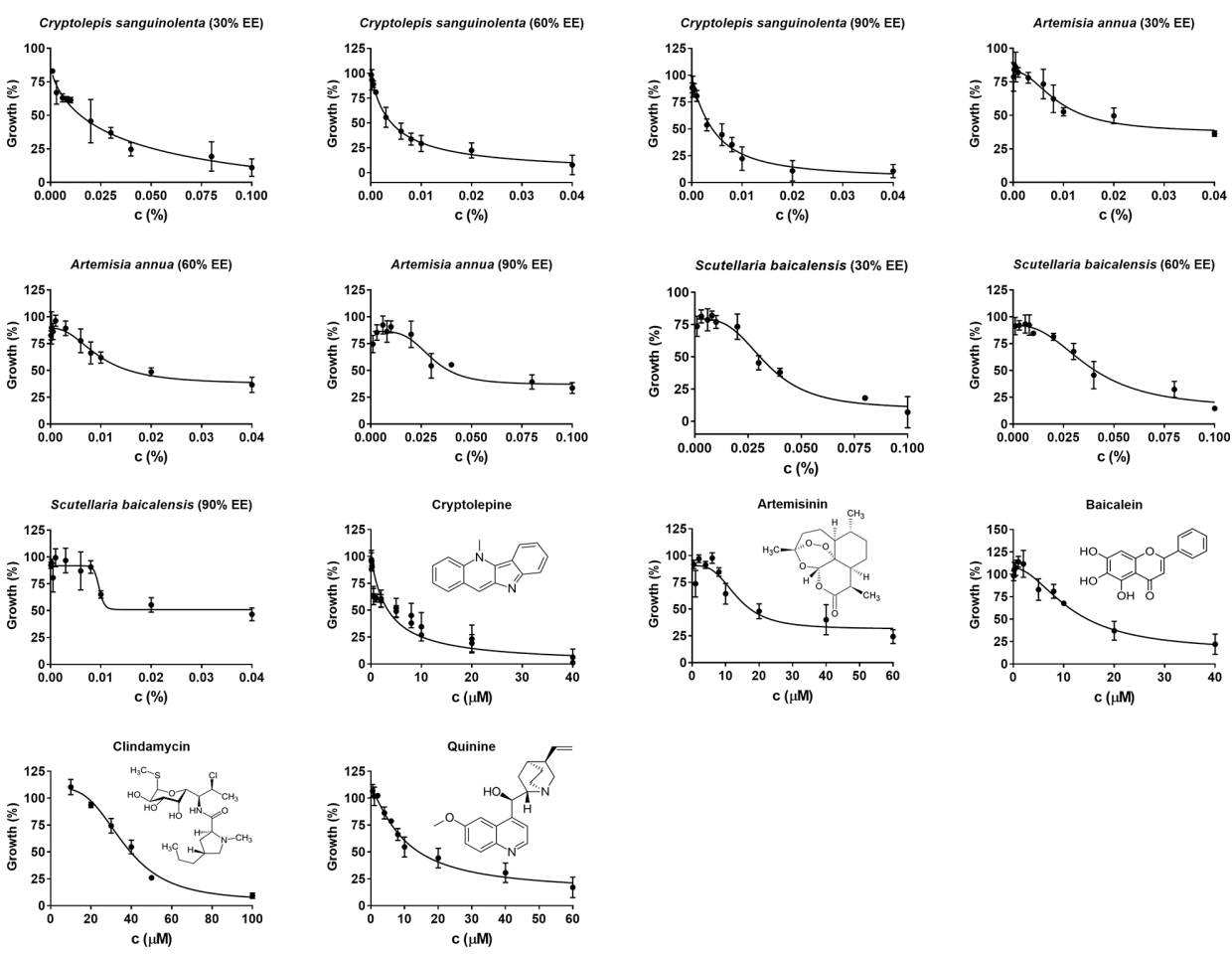

FIGURE 1 | Evaluation of in vitro natural products and drug compounds' susceptibility of B. duncani to Cryptolepis sanguilonlenta (30\%, 60\%, and 90\% EE), Artemisia annua (30\%, 60\%, and 90\% EE), Scutellaria baicalensis (30\%, 60\%, and 90\% EE), cryptolepine, artesunate, baicalein, clindamycin, and quinine at different concentrations. Each natural product or drug compound concentration was set in triplicate. SYBR Green I assay was performed at day three after exposure to the natural products or drug compounds. The growth of $B$. duncani in infected erythrocytes at day zero was set as $0 \%$. The growth of $B$. duncani in infected erythrocytes treated with 1\% DMSO vehicle at day three was set as 100\%. GraphPad Prism (version 7.0) was used to generate dose-response curves by fitting a nonlinear regression curve to the data. c, concentration; EE, ethanol extract. 


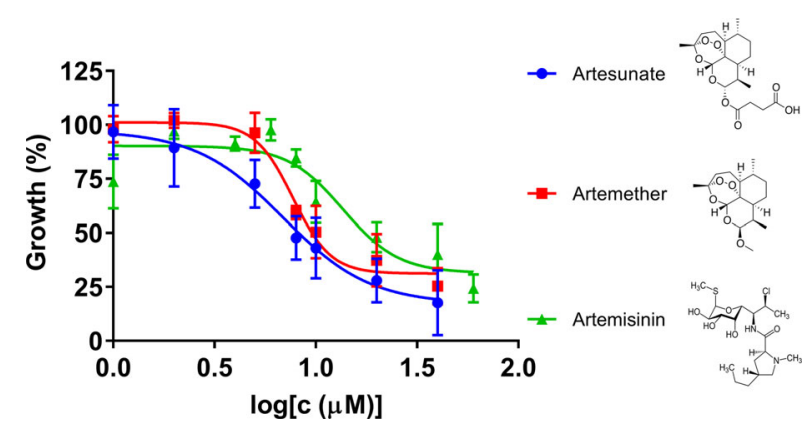

FIGURE 2 | The in vitro inhibitory activity of artesunate, artemether, and artemisinin against B. duncani at an initial parasitemia of $2 \%$ after three days incubation. The growth of $B$. duncani in infected erythrocytes at day zero was set as $0 \%$, and in the infected erythrocytes treated with $1 \%$ DMSO vehicle at day three was set as $100 \%$.

\section{Morphological Changes of Treated B. duncani}

After three days, the morphological changes of $B$. duncani treated with cryptolepine, artesunate, artemether, baicalein, clindamycin, and quinine at their respective concentrations of $1 \times, 2 \times, 4 \times \mathrm{IC}_{50}$ values were observed. The parasites treated by cryptolepine $\left(1 \times, 2 \times, 4 \times \mathrm{IC}_{50}\right.$ values $)$ appeared to have dramatically condensed chromatin in both trophozoite and schizont stages (Figure 3). Parasites exposed to artesunate, artemether, and clindamycin at $4 \times \mathrm{IC}_{50}$ values displayed teratogenic form, and those exposed to quinine showed gradually enlarged vacuole at the treated concentration of $1 \times$, $2 \times, 4 \times \mathrm{IC}_{50}$ values (Figure 3). B. duncani treated with artesunate $\left(1 \times, 2 \times \mathrm{IC}_{50}\right.$ values), artemether $\left(1 \times, 2 \times, \mathrm{IC}_{50}\right.$ values $)$, baicalein $\left(1 \times, 2 \times, 4 \times \mathrm{IC}_{50}\right.$ values $)$, and clindamycin $\left(1 \times, 2 \times \mathrm{IC}_{50}\right.$ values $)$ did not show significant morphological changes (Figure 3 ).

\section{Subculture Studies to Evaluate Viability of B. duncani Treated With Herb Extract Compounds Artesunate, Artemether, Cryptolepine, Baicalein, as Well as Quinine, and Clindamycin}

In order to further confirm our finding that cryptolepine, baicalein, artesunate, and artemether were the most active compounds for inhibiting $B$. duncani growth in vitro and that their corresponding natural product extracts indeed have a killing effect, we performed subculture studies by reinoculating three day treated infected erythrocytes into fresh uninfected erythrocytes to monitor regrowth. For comparison, we also evaluated the killing activity of quinine and clindamycin. The results showed that Artemisia annua (30\% ethanol extract) and Scutellaria baicalensis ( $90 \%$ ethanol extract) treated $B$. duncani could regrow at $1 \times, 2 \times, 4 \times$, and $8 \times \mathrm{IC}_{50}$ values, and growth of most of these treated parasites could be observed at day two (Figures 4A, B). Cryptolepis sanguinolenta (90\% ethanol extract) treated $B$. duncani regrew two days after treatment at $1 \times$ $\mathrm{IC}_{50}$ value, however, regrowth did not occur in six days after treatment at $2 \times, 4 \times$, and $8 \times \mathrm{IC}_{50}$ values (Figure $4 \mathrm{C}$ ). Similarly,
B. duncani treated with cryptolepine at $1 \times, 2 \times, 4 \times$, and $8 \times \mathrm{IC}_{50}$ values did not regrow in six days (Figure 4G). B. duncani treated with artesunate and artemether regrew after treatment at $1 \times, 2 \times$, and $4 \times \mathrm{IC}_{50}$ values, but no regrowth occurred after treatment with artemether at $8 \times \mathrm{IC}_{50}$ (Figures $\left.4 \mathrm{D}, \mathrm{E}\right)$. Treatment with baicalein at an $\mathrm{IC}_{50}$ of $1 \times$ and $2 \times$ did not prevent $B$. duncani regrowth at day two of incubation, and regrowth also occurred at day four when treated with baicalein at an $\mathrm{IC}_{50}$ of $4 \times$ and $8 \times$ (Figure 4F). The drug quinine showed good killing effect (Figure $\mathbf{4 H})$ at $1 \times, 2 \times, 4 \times$, and $8 \times \mathrm{IC}_{50}$ values, as did clindamycin at $2 \times$, $4 \times$, and $8 \times \mathrm{IC}_{50}$ values. However, $B$. duncani could regrow after clindamycin treatment at $1 \times \mathrm{IC}_{50}$ value (Figure $4 \mathrm{I}$ ).

\section{DISCUSSION}

While recovery from clinical babesiosis can be spontaneous in healthy individuals, there is a much greater risk of severe symptoms and fatality in elderly, asplenic, or otherwise immunocompromised patients (Vannier and Krause, 2012; Vannier et al., 2015). Furthermore, some patients can experience persistent infection and clinical illness despite treatment with current therapeutic regimens (Krause et al., 1998; Raffalli and Wormser, 2016; Bloch et al., 2019). Importantly, illness can be more severe and recalcitrant to available treatment regimens when patients are simultaneously infected with multiple tickborne pathogens (Krause et al., 2002; Horowitz and Freeman, 2019; Sanchez-Vicente et al., 2019). In addition, some physicians opine that patients infected with $B$. duncani can have a more protracted course and typically require a longer duration of treatment than those infected with $B$. microti (Scott and Scott, 2018). The combination of quinine and clindamycin is the treatment regimen recommended for $B$. duncani spp. infections (Vannier and Krause, 2009) and all severe babesiosis infections (Centers for Disease, 1983). However, a clinical trial indicated that $72 \%$ of patients who received quinine plus clindamycin for babesiosis suffered side effects including tinnitus, vertigo, and gastrointestinal upset, in some cases severe enough to necessitate dosage decrease or treatment suspension (Krause et al., 2000). Previous studies found that quinine showed $30-100 \mathrm{nM}$ of $\mathrm{EC}_{50}$ against Plasmodium falciparum (Skinner et al., 1996), and clindamycin showed around $8.8 \mathrm{nM}$ of $\mathrm{IC}_{50}$ against Plasmodium falciparum 3D7 (Dahl and Rosenthal, 2007). However, in this study we found that quinine and clindamycin had much higher $\mathrm{IC}_{50}$ values against $B$. duncani growth in hamster erythrocytes (10 $\mu \mathrm{M}$ and $37 \mu \mathrm{M}$, respectively), and these results are comparable with research done in vitro in human erythrocytes (Abraham et al., 2018). Our subculture results showed quinine had good killing effect in vitro in the range of $10-80 \mu \mathrm{M}$ (Figure $4 \mathrm{H}$ ), but relapse occurred after the treatment of clindamycin at the concentration of $37 \mu \mathrm{M}$ (Figure 4I). Importantly, we identified that the bioactive compounds derived from Cryptolepis sanguinolenta, Artemisia annua, and Scutellaria baicalensis, namely cryptolepine, artemisinin, artesunate, artemether, and baicalein $(3.4 \mu \mathrm{M}, 14 \mu \mathrm{M}, 7.4 \mu \mathrm{M}, 7.8 \mu \mathrm{M}$, and $12 \mu \mathrm{M}$, respectively) had comparable or even better activity against 


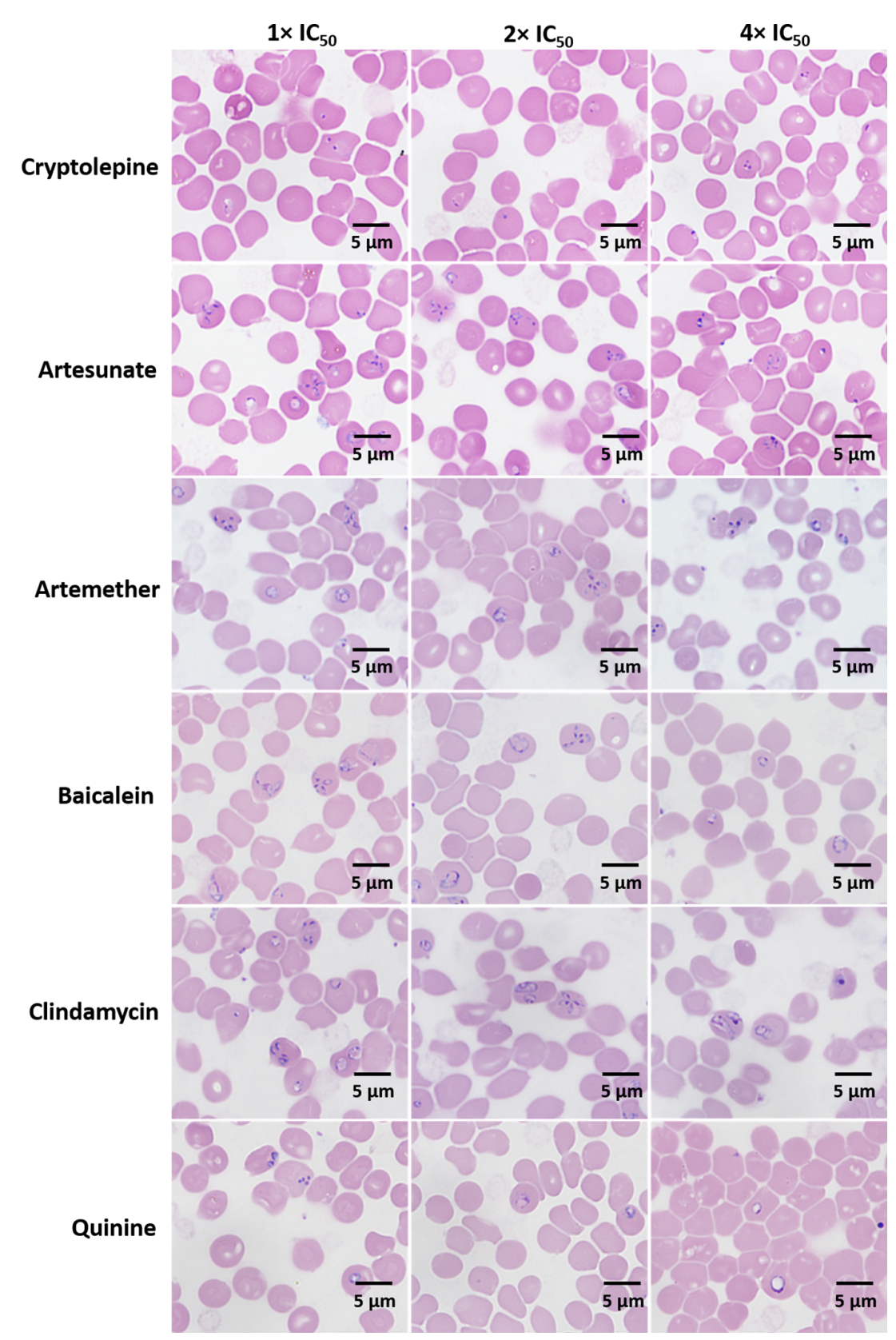

FIGURE 3 | Morphology of $B$. duncani observed after treatment with cryptolepine, artesunate, artemether, baicalein, clindamycin, and quinine at their respective concentrations of $1 \times, 2 \times$, and $4 \times 1 C_{50}$ values after three days of drug exposure.

B. duncani than the commonly used medications quinine $(10 \mu \mathrm{M})$ and clindamycin $(37 \mu \mathrm{M})$.

Botanical medicine has a long documented history of use over 5,000 years (Borchardt, 2002) and recent retrospective and systematic reviews have concluded severe adverse events associated with botanical medicine usage are rare (Di Lorenzo et al., 2015; Restani et al., 2016). Research over the past few decades has demonstrated that many botanical medicines have antimicrobial, antiparasitic, and anticancer activity (Tagboto and Townson, 2001; Shoemaker et al., 2005; Feng et al., 2011). In our previous study, we identified seven herbal medicines that have good activity against stationary phase Borrelia burgdorferi compared to the control antibiotics used in clinical treatment (Feng J. et al., 2020). In humans, Babesia spp. frequently exist as co-infections with Borrelia burgdorferi through tick transmission (Magnarelli et al., 1995; Parveen and Bhanot, 2019). The screening results of the present study revealed that Cryptolepis sanguinolenta, Artemisia annua, and Scutellaria baicalensis, which previously showed good activity against Borrelia burgdorferi (Tona et al., 1998), also had impressive inhibitory 

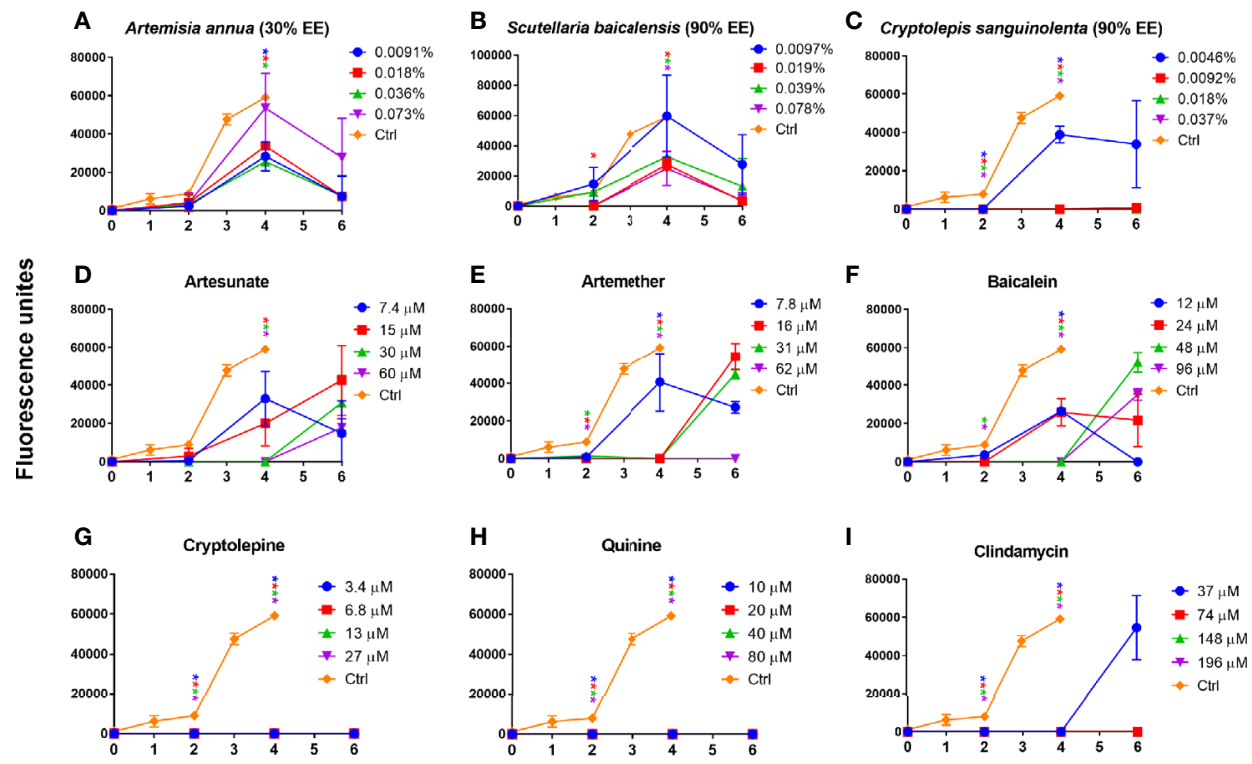

Days of Post-incubation

FIGURE 4 | In vitro subculture of B. duncani in hamster erythrocytes after three days exposure with Artemisia annua (A), Scutellaria baicalensis (B), Cryptolepis sanguinolenta (C), artesunate (D), artemether (E), baicalein (F), cryptolepine (G), quinine (H), and clindamycin (I) at their respective concentrations of $1 \times, 2 \times, 4 \times$, and $8 \times I_{50}$ values. The cultures treated with $1 \%$ DMSO vehicle were set as control. The growth of the subculture was examined by SYBR Green I assay, and the fluorescence units at day zero were normalized as zero. Asterisks indicate the significant difference $(p<0.05)$ between the respective treated group and the untreated control. EE, ethanol extract.

effects against B. duncani (at even lower concentrations of $0.01 \%)$. Additionally, we showed that herbs extracted by different concentrations of alcohol $(30 \%, 60 \%$, and $90 \%$ ethanol extracts) had different inhibitory activity. Extracts of Cryptolepis sanguinolenta and Scutellaria baicalensis in higher concentrations of alcohol ( $90 \%$ and $60 \%$ ethanol extracts) had better inhibitory activity than in low alcohol concentration $(30 \%$ ethanol extract), while Artemisia annua performed better at a lower alcohol concentration. This indicates that the corresponding bioactive ingredients of these herbs have diverse solubility in alcohol solvents, and that appropriate solvents and techniques are critical for the isolation and extraction of active constituents.

Cryptolepis sanguinolenta has been shown in preclinical studies to have anti-inflammatory, antimicrobial, anti-amoebic, anti-cancer, and anti-malarial properties (Grellier et al., 1996; Tona et al., 1998; Mills-Robertson et al., 2012; Ansah and Mensah, 2013; Hanprasertpong et al., 2014). Cryptolepine, an indoloquinoline alkaloid isolated from Cryptolepis sanguinolenta, has been shown in vitro to have significant antiplasmodial activity against drug-sensitive and drugresistant strains (Osafo et al., 2017; Ameyaw et al., 2018), demonstrating an $\mathrm{IC}_{50}$ of $0.2-0.6 \mu \mathrm{M}$ against Plasmodium falciparum in vitro (Grellier et al., 1996). In vivo, cryptolepine given orally at $50 \mathrm{mg} / \mathrm{kg} /$ day to mice infected with Plasmodium berghei was found to have moderate antimalarial activity to suppress parasitemia by $80 \%$ (Wright et al., 1996). The bioactivity of cryptolepine is believed to contribute to DNA intercalating activity targeting non-alternating CC sites, cell morphology change, and topoisomerase II inhibiting effects (Sawer et al., 1995; Bonjean et al., 1998; Lisgarten et al., 2002). Preliminary preclinical and clinical data indicate that Cryptolepis sanguinolenta and cryptolepine can have systemic effects in vivo and therefore are candidates for clinical development. Two openlabel trials using different formulations of Cryptolepis sanguinolenta showed significant efficacy without observed toxicity in the treatment of patients with uncomplicated malaria (Bugyei et al., 2010; Tempesta, 2010). The pharmacokinetics of cryptolepine revealed that it has low bioavailability, a moderate half-life (4.5h) and extensive distribution (Forkuo et al., 2017a). A nanoformulation of cryptolepine exhibited a more favorable pharmacokinetic profile and a stronger antiplasmodial effect compared to the unaltered form of cryptolepine (Forkuo et al., 2017b). Toxicology data suggests that Cryptolepis sanguinolenta is generally safe at doses under $500 \mathrm{mg} / \mathrm{kg}$ of body weight (Osafo et al., 2017), however further studies are needed to evaluate concerns regarding potential anti-fertility and embryotoxic effects (Ajayi and Akhigbe, 2012; Mensah et al., 2019). Recently, Batiha et al. demonstrated that cryptolepine exhibited significant activity against multiple Babesia spp. in vitro and inhibited B. microti in mice at a dose of $5 \mathrm{mg} / \mathrm{kg}$ (Batiha et al., 2020b). To the best of our knowledge no study has reported the in vitro or in vivo treatment efficacy of whole herb Cryptolepis sanguinolenta, against Babesia spp. Among the panel of natural product extracts tested in this study, we found Cryptolepis 
sanguinolenta extract had the strongest inhibitory activity against $B$. duncani in vitro and no regrowth was visible after 6 days of subculture at doses of $2 \times, 4 \times$, and $8 \times \mathrm{IC}_{50}$ values. We further confirmed that cryptolepine is one important active constituent with a nearly three-fold lower $\mathrm{IC}_{50}$ value against $B$. duncani than quinine, and ten-fold lower than clindamycin. Importantly, the non-viability of $B$. duncani treated with cryptolepine at a concentration as low as $1 \times \mathrm{IC}_{50}$ value (3.4 $\mu \mathrm{M}$; Figure 4G) further suggests that cryptolepine may be a promising compound to treat human babesiosis. Further studies are needed to confirm this.

The Chinese herb, Artemisia annua (Sweet wormwood or Qing Hao) has been used for the treatment of malaria for centuries (Feng X. et al., 2020) and the Chinese scientist who isolated its most famous active constituent, artemisinin, was awarded the Nobel Prize in 2015 in recognition for artemisinin's role in reducing malaria-associated morbidity and mortality (Liu and Liu, 2016). Artemisinin-based compounds have become pivotal in malaria treatment as they can reduce malarial parasitemia more rapidly than other known antimalarial drugs and are effective against all stages of Plasmodium spp (Meshnick et al., 1996; Olliaro et al., 2001). The mechanisms of action of artemisinin compounds are incompletely understood (Krause et al., 2007), although it has been proposed to be related to their ability to generate free radicals which can damage pathogen DNA and proteins (Eckstein-Ludwig et al., 2003; Wang et al., 2015). The safety, tolerability, and systemic effects of artemisinin-based compounds have been well-documented over decades of malarial clinical trials (Price et al., 1999; Adjuik et al., 2004; World Health Organization (WHO), 2015) including treatment during the $2^{\text {nd }}$ and $3^{\text {rd }}$ trimesters of pregnancy (Kovacs et al., 2016) and possibly during the first trimester (D'Alessandro et al., 2020). The safety, tolerability, and systemic effects of the whole herb, Artemisia annua, have also been documented, although in more limited clinical studies (Hunt et al., 2016, Stebbings et al., 2016; Yang et al., 2017). Babesia spp. and Plasmodium spp. share a similar intraerythrocytic life cycle and pathobiology which partly explains why artemisinin and its derivatives were able to inhibit in vitro or in vivo growth of $B$. gibsoni, B. equi, B. bigemina, B. bovis, and B. microti (Kumar et al., 2003; Goo et al., 2010; Mazuz et al., 2013; Iguchi et al., 2015). In our study, we found both $30 \%$ and $60 \%$ ethanol extracts of Artemisia annua had good inhibitory activity against $B$. duncani in vitro. The $\mathrm{IC}_{50}$ values of $30 \%, 60 \%$, and $90 \%$ ethanol extracts of Artemisia annua were 0.0091\%, 0.0097\%, and $0.030 \%$, respectively. A previous study indicated that flavonoids from Artemisia annua have potential synergistic anti-malaria effects with artemisinin (Ferreira et al., 2010). Because the content of artemisinin in the Artemisia annua samples used for extraction in this study is low $(0.11 \%$ as tested by high-performance liquid chromatography/UV-visual spectroscopy at the Institute for Food Safety and Defense, Centralia, WA), the role of anti-Babesia effects of other bioactive constituents such as flavonoids in Artemisia annua should not be ignored. Indeed, Artemisia spp. have documented antiparasitic effects beyond their artemisinin content. Artemisia afra, a non-artemisinin-containing species, was recently found to have similar efficacy as Artemisia annua, an artemisinincontaining species, in the treatment of malaria and schistosomiasis (Munyangi et al., 2018; Munyangi et al., 2019). Our study indicated that artemisinin and artemisinin derivatives (artesunate and artemether) alone also had in vitro inhibitory effects against B. duncani. Specifically, we found the $\mathrm{IC}_{50}$ values of artesunate and artemether were lower than that of quinine and clindamycin. It has been reported that B. gibsoni (wild type) was more susceptible to artemisinin than artesunate and artemether. Interestingly, the present study showed artesunate and artemether were more effective than artemisinin against $B$. duncani (Figure 2), and the $\mathrm{IC}_{50}$ values of artemisinin, artesunate, and artemether in this study were much higher than their corresponding $\mathrm{IC}_{50}$ values (nanomolar) in vitro against B. gibsoni (Matsuu et al., 2008; Iguchi et al., 2015). A previous study showed that $1 \mu \mathrm{g} / \mathrm{ml}(0.5 \mu \mathrm{M}$, approximately) of artesunate was sufficient to eradicate $B$. equi in vitro which was not able to regrow four days after drug withdrawal (Nagai et al., 2003). In contrast, our subculture study showed the regrowth of $B$. duncani could occur six days after treatment of artesunate at as high as $60 \mu \mathrm{M}\left(8 \times \mathrm{IC}_{50}\right)$, and of artesunate at $31 \mu \mathrm{M}\left(4 \times \mathrm{IC}_{50}\right)$. Because there is limited data in the research literature on the efficacy of artemisinin-related drugs against other Babesia spp., it remains unknown whether the low susceptibility to artemisinin, artesunate, and artemether is specific to B. duncani. Preliminary animal data suggest that adding a second anti-parasitic agent, such as cryptolepine from Cryptolepis sanguinolenta, may increase the susceptibility of artemisinin-based therapy to Babesia spp., similar to the use of two or three agents in artemisinin-based combination therapy (ACT) for malaria (Kumar et al., 2003; Iguchi et al., 2015; Forkuo et al., 2016; Carvalho et al., 2020). The development of malarial resistance to ACT raises important points regarding the potential benefits of using a whole plant form (e.g., Artemisia annua) vs a single constituent form (e.g., artemisinin) (Haldar et al., 2018). Although theoretic concerns that Artemisia annua's low artemisinin content may increase the risk of antimicrobial resistance (W.H.O.W.P.S, 2012) subsequent testing has revealed that antimicrobial resistance occurred three times slower when a whole plant form of Artemisia annua was used compared to artemisinin monotherapy (Elfawal et al., 2015). Additionally, preliminary clinical studies have revealed that treatment with whole plant Artemisia annua was associated with improved clinical outcomes and fewer side effects compared to ACT (Daddy et al., 2017; Munyangi et al., 2019). The improved outcomes when using a whole plant form of Artemisia annua can be explained by an evolutionarily guided synergism among its phytochemicals resulting in increased bioavailability of active compounds (pharmacodynamic synergy), complementary mechanisms of action (antimicrobial, immune-modulating, anti-inflammatory, etc.), multidrug resistance inhibition, and multiple antimicrobial compounds (Rasoanaivo et al., 2011; Munyangi et al., 2019; Caesar and Cech, 2019; Desrosiers et al., 2020, Zhao et al., 2020).

Scutellaria baicalensis (Chinese skullcap) has been widely used as a medicinal plant in China for thousands of years for 
the treatment of diarrhea, dysentery, hypertension, hemorrhage, insomnia, inflammation and respiratory infections (Zhao et al., 2016). Over forty different compounds have been isolated from Scutellaria baicalensis and the antimicrobial activity is hypothesized to be due to flavonoids, volatile oils, terpenoids, and polysaccharides (Wu et al., 2008; Lu et al., 2011; Leung et al., 2016). Previous studies have demonstrated that Scutellaria baicalensis extract and one of its primary bioactive constituents, baicalein, exhibited in vitro activity against various morphologic forms of Borrelia burgdorferi and Borrelia garinii (Goc et al., 2015; Feng J. et al., 2020). In traditional Korean medicine, Scutellaria baicalensis was combined with Artemisia apiacea for the treatment of malaria and other malaria-like diseases (Trinh et al., 2018). Existing clinical trial data on Scutellaria baicalensis indicates it is non-toxic and has systemic effects showing benefits in diabetes, enterovirus encephalitis, and adjunctive cancer care (Smol'ianinov et al., 1997; Lin et al., 2016; Shin et al., 2020). A medical food combination of concentrated baicalin and catechin (Limbrel ${ }^{\mathrm{TM}}$, Move Free Advanced ${ }^{\mathrm{TM}}$ ) was linked to reversible liver damage in at least 35 cases (Chalasani et al., 2012), however a recent study found no hepatotoxicity in patients taking a whole herb formulation of Scutellaria baicalensis dosed at 1,335 $\mathrm{mg}$ per day for an average of 444 days (Puri et al., 2019). Human safety studies on baicalin have shown that doses between $200-800 \mathrm{mg}$ per day are safe and well-tolerated with a favorable pharmacokinetic profile (Li et al., 2014; Pang et al., 2016). The anti-babesial activity of Scutellaria baicalensis and its active constituents have not been previously reported. In this study, we found Scutellaria baicalensis and baicalein showed good in vitro activity against $B$. duncani, with the $\mathrm{IC}_{50}$ value of baicalein practically the same as quinine $(12 \mu \mathrm{M}$ vs $10 \mu \mathrm{M})$, and three times lower than clindamycin $(12 \mu \mathrm{M}$ vs $37 \mu \mathrm{M})$. A study of ethanol extracts from Scutellaria baicalensis showed that it had the strongest inhibitory and bactericidal efficacy against six foodborne pathogens and had the highest content of flavonoids and phenolic acids (Lu et al., 2011). These findings are consistent with the present study which demonstrated Scutellaria baicalensis extract in $90 \%$ ethanol had a lower $\mathrm{IC}_{50}$ against $B$. duncani than those in $30 \%$ and $60 \%$ ethanol. Similar to artesunate, regrowth of B. duncani treated with baicalein did not occur in four days at $4 \times$ and $8 \times \mathrm{IC}_{50}$ values.

It should be noted that Alchornea cordifolia and Polygonum cuspidatum extracts also showed good inhibitory effect against $B$. duncani in our study. Both have been documented to have antimicrobial and anti-inflammatory activity (Ebi, 2001; Manga et al., 2004; Shan et al., 2008; Ghanim et al., 2010). Alchornea cordifolia has been used by traditional herbalists in several African countries for the treatment of malaria (Boniface et al., 2016) and pre-clinical studies corroborate significant antimalarial effects (Mustofa et al., 2000; Mesia et al., 2008; Ayisi et al., 2011). The active constituents of Alchornea cordifolia extract are complex, including phenolic acid, gallic acid, protocatechuic acid, ellagic acid, and quercetin (Boniface et al., 2016). Of note, ellagic acid has previously shown in vitro antiplasmodial activity (Lamikanra et al., 1990; Banzouzi et al.,
2002) and in vivo antibabesial activity (Beshbishy et al., 2019) and quercetin has been shown to have antiplasmodial and antibabesial effects (Batiha et al., 2020a). Although clinical data is lacking, preclinical studies reveal Alchornea cordifolia has favorable toxicology and bioavailability profiles (Gatsing et al., 2010; Ajibade and Olayemi, 2015; Boniface et al., 2016; Djimeli et al., 2017). Polygonum cuspidatum contains over 60 active constituents with wide-ranging mechanisms of action and varying degrees of bioavailability (Pan et al., 2019). Stilbenes (e.g., resveratrol) and hydroxyanthraquinones (e.g., emodin) are among the active constituents of Polygonum cuspidatum that exert antibacterial properties (Shan et al., 2008). The bioavailability of orally administered resveratrol and emodin is suboptimal (Walle, 2011; Dong et al., 2016) and therefore numerous strategies have been developed to improve their pharmacokinetic profiles (Chimento et al., 2019; Dewanjee et al., 2020). Despite its low bioavailability, resveratrol has high bioactivity which is thought to be related to extensive biotransformation in enterocytes, hepatocytes, and the gastrointestinal microbiome (Luca et al., 2020). Although various active constituents from Polygonum cuspidatum have been shown to have antiparasitic effects (Passos et al., 2015; Lin et al., 2017), to our knowledge, this is the first time that the antibabesial effects of Polygonum cuspidatum have been reported. Interestingly, our previous study indicated that Cryptolepis sanguinolenta, Artemisia annua, Scutellaria baicalensis, and Polygonum cuspidatum extracts have activity against Borrelia burgdorferi (Feng J. et al., 2020), indicating that it may be advantageous to use these herbs to simultaneously target these different pathogens in complex Lyme disease with co-infections.

A potential limitation of the study is the descriptive nature of the study. Future studies are needed to determine the active components of the effective hits, to better investigate their effects on Babesia morphology through microscopy techniques, and to characterize if extracts produce Babesia death or just inhibit its growth or cell cycle, and to confirm their activity in animal models.

\section{CONCLUSIONS}

In this study we identified herbal medicines Cryptolepis sanguinolenta, Artemisia annua, Scutellaria baicalensis, Alchornea cordifolia, and Polygonum cuspidatum that exhibit good in vitro inhibitory activity against B. duncani at $0.01 \%(\mathrm{v} / \mathrm{v})$. Furthermore, we tested the activity of the potential bioactive constituents and found that cryptolepine, artemisinin-related compounds, and baicalein may be promising alternatives to treat human babesiosis caused by $B$. duncani. These botanical medicines can be considered candidates for clinical development based on their low cost, favorable toxicity and pharmacokinetic profiles and history of safety and efficacy from clinical trials and/ or use in traditional medicine. Future research will be needed to identify the bioactive components and to test if they also have activity against other Babesia species in vitro and in vivo. In order to identify more effective treatments, combinations of these 
active herbs and active compounds need to be tested both in vitro and in animal models. This research needs to be done both in isolation and potentially in combination with current antibabesial drugs. Since the botanical medicines Cryptolepis sanguinolenta, Artemisia annua, Scutellaria baicalensis, Alchornea cordifolia, and Polygonum cuspidatum are already in clinical use, it is also important for future studies to evaluate them directly in patients using specific clinical treatment regimens.

\section{DATA AVAILABILITY STATEMENT}

The original contributions presented in the study are included in the article/Supplementary Material. Further inquiries can be directed to the corresponding author.

\section{ETHICS STATEMENT}

The animal study was reviewed and approved by Johns Hopkins Institutional Animal Care and Use Committee.

\section{AUTHOR CONTRIBUTIONS}

Conceptualization, YMZ and YZ. Methodology, YMZ, YZ, HA-M, and JL. Data curation, YMZ and HA-M. Funding acquisition, YZ. Writing-original draft, YMZ. Writing-

\section{REFERENCES}

Abraham, A., Brasov, I., Thekkiniath, J., Kilian, N., Lawres, L., Gao, R., et al. (2018). Establishment of a continuous in vitro culture of Babesia duncani in human erythrocytes reveals unusually high tolerance to recommended therapies. J. Biol. Chem. 293, 19974-19981. doi: 10.1074/jbc.AC118.005771

Adjuik, M., Babiker, A., Garner, P., Olliaro, P., Taylor, W., White, N., et al. (2004). Artesunate combinations for treatment of malaria: meta-analysis. Lancet 363, 9-17. doi: 10.1016/S0140-6736(03)15162-8

Ajayi, A. F., and Akhigbe, R. E. (2012). Antifertility activity of Cryptolepis sanguinolenta leaf ethanolic extract in male rats. J. Hum. Reprod. Sci. 5, 4347. doi: 10.4103/0974-1208.97799

Ajibade, T. O., and Olayemi, F. O. (2015). Reproductive and toxic effects of methanol extract of Alchornea cordifolia leaf in male rats. Andrologia 47, 10341040. doi: 10.1111/and.12374

Ameyaw, E. O., Asmah, K. B., Biney, R. P., Henneh, I. T., Owusu-Agyei, P., Prah, J., et al. (2018). Isobolographic analysis of co-administration of two plant-derived antiplasmodial drug candidates, cryptolepine and xylopic acid, in Plasmodium berghei. Malar. J. 17, 153. doi: 10.1186/s12936-018-2283-8

Ansah, C., and Mensah, K. B. (2013). A review of the anticancer potential of the antimalarial herbal Cryptolepis sanguinolenta and its major alkaloid cryptolepine. Ghana Med. J. 47, 137-147.

Ayisi, N. K., Appiah-Opong, R., Gyan, B., Bugyei, K., and Ekuban, F. (2011). Plasmodium falciparum: Assessment of Selectivity of Action of Chloroquine, Alchornea cordifolia, Ficus polita, and Other Drugs by a Tetrazolium-Based Colorimetric Assay. Malar. Res. Treat 2011, 816250. doi: 10.4061/2011/816250

Banzouzi, J. T., Prado, R., Menan, H., Valentin, A., Roumestan, C., Mallie, M., et al. (2002). In vitro antiplasmodial activity of extracts of Alchornea cordifolia and identification of an active constituent: ellagic acid. J. Ethnopharmacol. 81, 399401. doi: 10.1016/S0378-8741(02)00121-6 review and editing, JL, SS, and YZ. All authors contributed to the article and approved the submitted version.

\section{FUNDING}

Sources of funding included Bay Area Lyme Foundation, the Steven and Alexandra Cohen Foundation, Global Lyme Alliance, NatCapLyme, LivLyme Foundation, and the Einstein-Sim Family Charitable Fund.

\section{ACKNOWLEDGMENTS}

We gratefully acknowledge the generous support of Bay Area Lyme Foundation, the Steven and Alexandra Cohen Foundation, Global Lyme Alliance, NatCapLyme, LivLyme Foundation, and the Einstein-Sim Family Charitable Fund. We thank BEI Resources for providing the Babesia duncani strain WA1 (ATCC $^{\circledR}$ PRA-302 ${ }^{\mathrm{TM}}$ ) used in this study. We thank herbalists Eric Yarnell ND and Brian Kie Weissbuch for providing some of the botanical extracts for evaluation in this study.

\section{SUPPLEMENTARY MATERIAL}

The Supplementary Material for this article can be found online at: https://www.frontiersin.org/articles/10.3389/fcimb.2021. 624745/full\#supplementary-material

Batiha, G. E., Beshbishy, A. M., Ikram, M., Mulla, Z. S., El-Hack, M. E. A., Taha, A. E., et al. (2020a). The Pharmacological Activity, Biochemical Properties, and Pharmacokinetics of the Major Natural Polyphenolic Flavonoid: Quercetin. Foods 9. doi: 10.3390/foods9030374

Batiha, G. E., Beshbishy, A. M., Alkazmi, L. M., Nadwa, E. H., Rashwan, E. K., Yokoyama, N., et al. (2020b). In vitro and in vivo growth inhibitory activities of cryptolepine hydrate against several Babesia species and Theileria equi. PLoS. Negl. Trop. Dis. 14 (8), e0008489. doi: 10.1371/journal.pntd.0008489

Beshbishy, A. M., Batiha, G. E., Yokoyama, N., and Igarashi, I. (2019). Ellagic acid microspheres restrict the growth of Babesia and Theileria in vitro and Babesia microti in vivo. Parasit. Vectors 12, 269. doi: 10.1186/s13071-019-3520-x

Birkenheuer, A. J., Whittington, J., Neel, J., Large, E., Barger, A., Levy, M. G., et al. (2006). Molecular characterization of a Babesia species identified in a North American raccoon. J. Wildl. Dis. 42, 375-380. doi: 10.7589/0090-3558-42.2.375

Bloch, E. M., Kumar, S., and Krause, P. J. (2019). Persistence of Babesia microti Infection in Humans. Pathogens 8 (3), 102. doi: 10.3390/pathogens8030102

Boniface, P. K., Ferreira, S. B., and Kaiser, C. R. (2016). Recent trends in phytochemistry, ethnobotany and pharmacological significance of Alchornea cordifolia (Schumach. \& Thonn.) Muell. Arg. J. Ethnopharmacol. 191, 216-244. doi: 10.1016/j.jep.2016.06.021

Bonjean, K., De Pauw-Gillet, M. C., Defresne, M. P., Colson, P., Houssier, C., Dassonneville, L., et al. (1998). The DNA intercalating alkaloid cryptolepine interferes with topoisomerase II and inhibits primarily DNA synthesis in B16 melanoma cells. Biochemistry 37, 5136-5146. doi: 10.1021/bi972927q

Borchardt, J. K. (2002). The Beginnings of Drug Therapy: Ancient Mesopotamian Medicine. Drug News Perspect. 15, 187-192. doi: 10.1358/dnp.2002. 15.3.840015

Bugyei, K. A., Boye, G. L., and Addy, M. E. (2010). Clinical efficacy of a tea-bag formulation of Cryptolepis sanguinolenta root in the treatment of acute uncomplicated falciparum malaria. Ghana Med. J. 44, 3-9. doi: 10.4314/ gmj.v44i1.68849 
Buhner, S. H. (2015). Natural Treatments For Lyme Coinfections: Anaplasma, Babesia, And Ehrlichia (Rochester, Vermont: Healing Arts Press).

Caesar, L. K., and Cech, N. B. (2019). Synergy and antagonism in natural product extracts: when $1+1$ does not equal 2. Nat. Prod. Rep. 36, 869-888. doi: 10.1039/C9NP00011A

Carvalho, L. J. M., Tuvshintulga, B., Nugraha, A. B., Sivakumar, T., and Yokoyama, N. (2020). Activities of artesunate-based combinations and tafenoquine against Babesia bovis in vitro and Babesia microti in vivo. Parasit. Vectors 13, 362. doi: 10.1186/s13071-020-04235-7

Centers for Disease Control (CDC). (1983). Clindamycin and quinine treatment for Babesia microti infections. MMWR Morb. Mortal. Wkly. Rep. 32, 65-6, 72.

Chalasani, N., Vuppalanchi, R., Navarro, V., Fontana, R., Bonkovsky, H., Barnhart, H., et al. (2012). Acute Liver Injury due to Flavocoxid (Limbrel), a Medical Food for Osteoarthritis. Ann. Internal Med. 156, 857-U129. doi: 10.7326/0003-4819-156-12-201206190-00006

Chimento, A., De Amicis, F., Sirianni, R., Sinicropi, M. S., Puoci, F., Casaburi, I., et al. (2019). Progress to Improve Oral Bioavailability and Beneficial Effects of Resveratrol. Int. J. Mol. Sci. 20 (6), 1381. doi: 10.3390/ijms20061381

Cimanga, K., De Bruyne, T., Lasure, A., Van Poel, B., Pieters, L., Claeys, M., et al. (1996). In vitro biological activities of alkaloids from Cryptolepis sanguinolenta. Planta Med. 62, 22-27. doi: 10.1055/s-2006-957789

Daddy, N. B., Kalisya, L. M., Bagire, P. G., Watt, R. L., Towler, M. J., and Weathers, P. J. (2017). Artemisia annua dried leaf tablets treated malaria resistant to ACT and i.v. artesunate: Case reports. Phytomedicine 32, 37-40. doi: 10.1016/ j.phymed.2017.04.006

Dahl, E. L., and Rosenthal, P. J. (2007). Multiple antibiotics exert delayed effects against the Plasmodium falciparum apicoplast. Antimicrob. Agents Chemother. 51, 3485-3490. doi: 10.1128/AAC.00527-07

Desrosiers, M. R., Mittelman, A., and Weathers, P. J. (2020). Dried Leaf Artemisia Annua Improves Bioavailability of Artemisinin via Cytochrome P450 Inhibition and Enhances Artemisinin Efficacy Downstream. Biomolecules 10 (2), 254. doi: 10.3390/biom 10020254

Dewanjee, S., Chakraborty, P., Mukherjee, B., and De Feo, V. (2020). Plant-Based Antidiabetic Nanoformulations: The Emerging Paradigm for Effective Therapy. Int. J. Mol. Sci. 21 (6), 2217. doi: 10.3390/ijms21062217

Di Lorenzo, C., Ceschi, A., Kupferschmidt, H., Lude, S., De Souza Nascimento, E., Dos Santos, A., et al. (2015). Adverse effects of plant food supplements and botanical preparations: a systematic review with critical evaluation of causality. Br. J. Clin. Pharmacol. 79, 578-592. doi: 10.1111/bcp.12519

Diuk-Wasser, M. A., Vannier, E., and Krause, P. J. (2016). Coinfection by Ixodes Tick-Borne Pathogens: Ecological, Epidemiological, and Clinical Consequences. Trends Parasitol. 32, 30-42. doi: 10.1016/j.pt.2015.09.008

Djimeli, M. N., Fodouop, S. P. C., Njateng, G. S. S., Fokunang, C., Tala, D. S., Kengni, F., et al. (2017). Antibacterial activities and toxicological study of the aqueous extract from leaves of Alchornea cordifolia (Euphorbiaceae). BMC Complement. Altern. Med. 17, 349. doi: 10.1186/s12906-017-1854-5

Dong, X., Fu, J., Yin, X., Cao, S., Li, X., Lin, L., et al. (2016). Emodin: A Review of its Pharmacology, Toxicity and Pharmacokinetics. Phytother. Res. 30, 12071218. doi: $10.1002 /$ ptr.5631

D’Alessandro, S., Menegola, E., Parapini, S., Taramelli, D., and Basilico, N. (2020). Safety of Artemisinin Derivatives in the First Trimester of Pregnancy: A Controversial Story. Molecules 25 (15), 3505. doi: 10.3390/molecules25153505

Ebi, G. C. (2001). Antimicrobial activities of Alchornea cordifolia. Fitoterapia 72, 69-72. doi: 10.1016/S0367-326X(00)00254-9

Eckstein-Ludwig, U., Webb, R. J., Van Goethem, I. D., East, J. M., Lee, A. G., Kimura, M., et al. (2003). Artemisinins target the SERCA of Plasmodium falciparum. Nature 424, 957-961. doi: 10.1038/nature01813

Elfawal, M. A., Towler, M. J., Reich, N. G., Weathers, P. J., and Rich, S. M. (2015). Dried whole-plant Artemisia annua slows evolution of malaria drug resistance and overcomes resistance to artemisinin. Proc. Natl. Acad. Sci. U.S.A. 112, 821826. doi: $10.1073 /$ pnas. 1413127112

Feng, Y., Wang, N., Zhu, M., Feng, Y., Li, H., and Tsao, S. (2011). Recent progress on anticancer candidates in patents of herbal medicinal products. Recent Pat. Food Nutr. Agric. 3, 30-48. doi: 10.2174/2212798411103010030

Feng, J., Leone, J., Schweig, S., and Zhang, Y. (2020). Evaluation of Natural and Botanical Medicines for Activity Against Growing and Non-growing Forms of B. Burgdorferi. Front. Med. (Lausanne) 7, 6. doi: 10.3389/fmed.2020.00006
Feng, X., Cao, S., Qiu, F., and Zhang, B. (2020). Traditional application and modern pharmacological research of Artemisia annua L. Pharmacol. Ther., 16, 107650. doi: 10.1016/j.pharmthera.2020.107650

Ferreira, J. F. S., Luthria, D. L., Sasaki, T., and Heyerick, A. (2010). Flavonoids from Artemisia annua L. as Antioxidants and Their Potential Synergism with Artemisinin against Malaria and Cancer. Molecules 15, 3135-3170. doi: 10.3390/molecules 15053135

Forkuo, A. D., Ansah, C., Boadu, K. M., Boampong, J. N., Ameyaw, E. O., Gyan, B. A., et al. (2016). Synergistic anti-malarial action of cryptolepine and artemisinins. Malar. J. 15, 89. doi: 10.1186/s12936-016-1137-5

Forkuo, A. D., Ansah, C., Pearson, D., Gertsch, W., Cirello, A., Amaral, A., et al. (2017a). Identification of cryptolepine metabolites in rat and human hepatocytes and metabolism and pharmacokinetics of cryptolepine in Sprague Dawley rats. BMC Pharmacol. Toxicol. 18, 84. doi: 10.1186/s40360017-0188-8

Forkuo, A. D., Ansah, C., Mensah, K. B., Annan, K., Gyan, B., Theron, A., et al. (2017b). In vitro anti-malarial interaction and gametocytocidal activity of cryptolepine. Malar. J. 16, 496. doi: 10.1186/s12936-017-2142-z

Gatsing, D., Nkeugouapi, C. F. N., Nji-Nkah, B. F., Kuiate, J. R., and Tchouanguep, F. M. (2010). Antibacterial Activity, Bioavailability and Acute Toxicity Evaluation of the Leaf Extract of Alchornea cordifolia (Euphorbiaceae). Int. J. Pharmacol. 6, 173-182. doi: 10.3923/ijp.2010.173.182

Ghanim, H., Sia, C. L., Abuaysheh, S., Korzeniewski, K., Patnaik, P., Marumganti, A., et al. (2010). An antiinflammatory and reactive oxygen species suppressive effects of an extract of Polygonum cuspidatum containing resveratrol. J. Clin. Endocrinol. Metab. 95, E1-E8. doi: 10.1210/mend.24.7.9998

Goc, A., Niedzwiecki, A., and Rath, M. (2015). In vitro evaluation of antibacterial activity of phytochemicals and micronutrients against Borrelia burgdorferi and Borrelia garinii. J. Appl. Microbiol. 119, 1561-1572. doi: 10.1111/jam.12970

Gonzalez, L. M., Rojo, S., Gonzalez-Camacho, F., Luque, D., Lobo, C. A., and Montero, E. (2014). Severe babesiosis in immunocompetent man, Spain, 2011. Emerg. Infect. Dis. 20, 724-726. doi: 10.3201/eid2004.131409

Goo, Y. K., Terkawi, M. A., Jia, H., Aboge, G. O., Ooka, H., Nelson, B., et al. (2010). Artesunate, a potential drug for treatment of Babesia infection. Parasitol. Int. 59, 481-486. doi: 10.1016/j.parint.2010.06.004

Grellier, P., Ramiaramanana, L., Millerioux, V., Deharo, E., Schrevel, J., Frappier, F., et al. (1996). Antimalarial activity of cryptolepine and isocryptolepine, alkaloids isolated from Cryptolepis sanguinolenta. Phytother. Res. 10, 317-321. doi: 10.1002/ (SICI)1099-1573(199606)10:4<317::AID-PTR858>3.0.CO;2-0

Haldar, K., Bhattacharjee, S., and Safeukui, I. (2018). Drug resistance in Plasmodium. Nat. Rev. Microbiol. 16, 156-170. doi: 10.1038/nrmicro.2017.161

Hanprasertpong, N., Teekachunhatean, S., Chaiwongsa, R., Ongchai, S., Kunanusorn, P., Sangdee, C., et al. (2014). Analgesic, anti-inflammatory, and chondroprotective activities of Cryptolepis buchanani extract: in vitro and in vivo studies. BioMed. Res. Int. 2014, 978582. doi: 10.1155/2014/978582

Horowitz, R. I., and Freeman, P. R. (2019). Precision medicine: retrospective chart review and data analysis of 200 patients on dapsone combination therapy for chronic Lyme disease/post-treatment Lyme disease syndrome: part 1. Int. J. Gen. Med. 12, 101-119. doi: 10.2147/IJGM.S193608

Hunt, S., Stebbings, S., and McNamara, D. (2016). An open-label six-month extension study to investigate the safety and efficacy of an extract of Artemisia annua for managing pain, stiffness and functional limitation associated with osteoarthritis of the hip and knee. N. Z. Med. J. 129, 97-102.

Iguchi, A., Matsuu, A., Matsuyama, K., and Hikasa, Y. (2015). The efficacy of artemisinin, artemether, and lumefantrine against Babesia gibsoni in vitro. Parasitol. Int. 64, 190-193. doi: 10.1016/j.parint.2014.12.006

Jaiswal, Y., Liang, Z., and Zhao, Z. (2016). Botanical drugs in Ayurveda and Traditional Chinese Medicine. J. Ethnopharmacol. 194, 245-259. doi: 10.1016/ j.jep.2016.06.052

Kjemtrup, A. M., and Conrad, P. A. (2000). Human babesiosis: an emerging tick-borne disease. Int. J. Parasitol. 30, 1323-1337. doi: 10.1016/S0020-7519(00)00137-5

Klayman, D. L. (1993). Artemisia annua - from Weed To Respectable Antimalarial Plant. ACS Sym. Ser. 534, 242-255. doi: 10.1021/bk-1993-0534.ch017

Kovacs, S. D., van Eijk, A. M., Sevene, E., Dellicour, S., Weiss, N. S., Emerson, S., et al. (2016). The Safety of Artemisinin Derivatives for the Treatment of Malaria in the 2nd or 3rd Trimester of Pregnancy: A Systematic Review and Meta-Analysis. PloS One 11, e0164963. doi: 10.1371/journal.pone.0164963 
Krause, P. J., Telford, S. R. 3., Spielman, A., Sikand, V., Ryan, R., Christianson, D., et al. (1996). Concurrent Lyme disease and babesiosis. Evidence for increased severity and duration of illness. JAMA 275, 1657-1660. doi: 10.1001/ jama.1996.03530450047031

Krause, P. J., Spielman, A., Telford, S. R. 3., Sikand, V. K., McKay, K., Christianson, D., et al. (1998). Persistent parasitemia after acute babesiosis. N. Engl. J. Med. 339, 160 165. doi: 10.1056/NEJM199807163390304

Krause, P. J., Lepore, T., Sikand, V. K., Gadbaw, J. Jr., Burke, G., Telford, S. R. 3., et al. (2000). Atovaquone and azithromycin for the treatment of babesiosis. $N$. Engl. J. Med. 343, 1454-1458. doi: 10.1056/NEJM200011163432004

Krause, P. J., McKay, K., Thompson, C. A., Sikand, V. K., Lentz, R., Lepore, T., et al. (2002). Disease-specific diagnosis of coinfecting tickborne zoonoses: babesiosis, human granulocytic ehrlichiosis, and Lyme disease. Clin. Infect. Dis. 34, 1184-1191. doi: 10.1086/339813

Krause, P. J., Daily, J., Telford, S. R., Vannier, E., Lantos, P., and Spielman, A. (2007). Shared features in the pathobiology of babesiosis and malaria. Trends Parasitol. 23, 605-610. doi: 10.1016/j.pt.2007.09.005

Krause, P. J., Gewurz, B. E., Hill, D., Marty, F. M., Vannier, E., Foppa, I. M., et al. (2008). Persistent and relapsing babesiosis in immunocompromised patients. Clin. Infect. Dis. 46, 370-376. doi: 10.1086/525852

Kumar, S., Gupta, A. K., Pal, Y., and Dwivedi, S. K. (2003). In-vivo therapeutic efficacy trial with artemisinin derivative, buparvaquone and imidocarb dipropionate against Babesia equi infection in donkeys. J. Vet. Med. Sci. 65, 1171-1177. doi: 10.1292/jvms.65.1171

Lamikanra, A., Ogundaini, A. O., and Ogungbamila, F. O. (1990). Antibacterial Constituents of Alchornea-Cordifolia Leaves. Phytother. Res. 4, 198-200. doi: 10.1002/ptr.2650040508

Leiby, D. A. (2006). Babesiosis and blood transfusion: flying under the radar. Vox. Sang. 90, 157-165. doi: 10.1111/j.1423-0410.2006.00740.x

Leung, K. C., Seneviratne, C. J., Li, X., Leung, P. C., Lau, C. B., Wong, C. H., et al. (2016). Synergistic Antibacterial Effects of Nanoparticles Encapsulated with Scutellaria baicalensis and Pure Chlorhexidine on Oral Bacterial Biofilms. Nanomater. (Basel) 6 (4), 61. doi: 10.3390/nano6040061

Li, M., Shi, A., Pang, H., Xue, W., Li, Y., Cao, G., et al. (2014). Safety, tolerability, and pharmacokinetics of a single ascending dose of baicalein chewable tablets in healthy subjects. J. Ethnopharmacol. 156, 210-215. doi: 10.1016/ j.jep.2014.08.031

Li, Y., Liu, M., Rizk, M. A., Moumouni, P. F. A., Lee, S. H., Galon, E. M., et al. (2020). Drug screening of food and drug administration-approved compounds against Babesia bovis in vitro. Exp. Parasitol. 210, 107831. doi: 10.1016/ j.exppara.2020.107831

Lin, H., Zhou, J., Lin, K., Wang, H., Liang, Z., Ren, X., et al. (2016). Efficacy of Scutellaria baicalensis for the Treatment of Hand, Foot, and Mouth Disease Associated with Encephalitis in Patients Infected with EV71: A Multicenter. Retrospect. Analysis BioMed. Res. Int. 2016, 5697571. doi: 10.1155/2016/ 5697571

Lin, B. C., Harris, D. R., Kirkman, L. M. D., Perez, A. M., Qian, Y. W., Schermerhorn, J. T., et al. (2017). FIKK Kinase, a Ser/Thr Kinase Important to Malaria Parasites, Is Inhibited by Tyrosine Kinase Inhibitors. ACS Omega 2, 6605-6612. doi: 10.1021/acsomega.7b00997

Lisgarten, J. N., Coll, M., Portugal, J., Wright, C. W., and Aymami, J. (2002). The antimalarial and cytotoxic drug cryptolepine intercalates into DNA at cytosine-cytosine sites. Nat. Struct. Biol. 9, 57-60. doi: 10.1038/nsb729

Liu, W., and Liu, Y. (2016). Youyou Tu: significance of winning the 2015 Nobel Prize in Physiology or Medicine. Cardiovasc. Diagn. Ther. 6, 1-2. doi: 10.3978/ j.issn.2223-3652.2015.12.11

Lu, Y., Joerger, R., and Wu, C. (2011). Study of the chemical composition and antimicrobial activities of ethanolic extracts from roots of Scutellaria baicalensis Georgi. J. Agric. Food Chem. 59, 10934-10942. doi: 10.1021/ jf202741x

Luca, S. V., Macovei, I., Bujor, A., Miron, A., Skalicka-Wozniak, K., Aprotosoaie, A. C., et al. (2020). Bioactivity of dietary polyphenols: The role of metabolites. Crit. Rev. Food Sci. Nutr. 60, 626-659. doi: 10.1080/10408398.2018.1546669

Lux, J. Z., Weiss, D., Linden, J. V., Kessler, D., Herwaldt, B. L., Wong, S. J., et al. (2003). Transfusion-associated babesiosis after heart transplant. Emerg. Infect. Dis. 9, 116-119. doi: 10.3201/eid0901.020149

Magnarelli, L. A., Dumler, J. S., Anderson, J. F., Johnson, R. C., and Fikrig, E. (1995). Coexistence of antibodies to tick-borne pathogens of babesiosis, ehrlichiosis, and Lyme borreliosis in human sera. J. Clin. Microbiol. 33, 3054-3057. doi: 10.1128/JCM.33.11.3054-3057.1995

Manga, H. M., Brkic, D., Marie, D. E., and Quetin-Leclercq, J. (2004). In vivo antiinflammatory activity of Alchornea cordifolia (Schumach. Thonn.) Mull. Arg. (Euphorbiaceae). J. Ethnopharmacol. 92, 209-214. doi: 10.1016/ j.jep.2004.02.019

Matsuu, A., Yamasaki, M., Xuan, X., Ikadai, H., and Hikasa, Y. (2008). In vitro evaluation of the growth inhibitory activities of 15 drugs against Babesia gibsoni (Aomori strain). Vet. Parasitol. 157, 1-8. doi: 10.1016/ j.vetpar.2008.07.023

Mazuz, M. L., Golenser, J., Fish, L., Haynes, R. K., Wollkomirsky, R., Leibovich, B., et al. (2013). Artemisone inhibits in vitro and in vivo propagation of Babesia bovis and B. bigemina parasites. Exp. Parasitol. 135, 690-694. doi: 10.1016/ j.exppara.2013.10.006

Mensah, K. B., Benneh, C., Forkuo, A. D., and Ansah, C. (2019). Cryptolepine, the Main Alkaloid of the Antimalarial Cryptolepis sanguinolenta (Lindl.) Schlechter, Induces Malformations in Zebrafish Embryos. Biochem. Res. Int 2019, 7076986. doi: 10.1155/2019/7076986

Meshnick, S. R., Taylor, T. E., and Kamchonwongpaisan, S. (1996). Artemisinin and the antimalarial endoperoxides: from herbal remedy to targeted chemotherapy. Microbiol. Rev. 60, 301-315. doi: 10.1128/MR.60.2.301315.1996

Mesia, G. K., Tona, G. L., Nanga, T. H., Cimanga, R. K., Apers, S., Cos, P., et al. (2008). Antiprotozoal and cytotoxic screening of 45 plant extracts from Democratic Republic of Congo. J. Ethnopharmacol. 115, 409-415. doi: 10.1016/j.jep.2007.10.028

Mills-Robertson, F. C., Tay, S. C., Duker-Eshun, G., Walana, W., and Badu, K. (2012). In vitro antimicrobial activity of ethanolic fractions of Cryptolepis sanguinolenta. Ann. Clin. Microbiol. Antimicrob. 11, 16. doi: 10.1186/14760711-11-16

Moro, M. H., David, C. S., Magera, J. M., Wettstein, P. J., Barthold, S. W., and Persing, D. H. (1998). Differential effects of infection with a Babesia-like piroplasm, WA1, in inbred mice. Infect. Immun. 66, 492-498. doi: 10.1128/ IAI.66.2.492-498.1998

Munyangi, J., Cornet-Vernet, L., Idumbo, M., Lu, C., Lutgen, P., Perronne, C., et al. (2018). Effect of Artemisia annua and Artemisia afra tea infusions on schistosomiasis in a large clinical trial. Phytomedicine 51, 233-240. doi: 10.1016/j.phymed.2018.10.014

Munyangi, J., Cornet-Vernet, L., Idumbo, M., Lu, C., Lutgen, P., Perronne, C., et al. (2019). Artemisia annua and Artemisia afra tea infusions vs. artesunateamodiaquine (ASAQ) in treating Plasmodium falciparum malaria in a large scale, double blind, randomized clinical trial. Phytomedicine 57, 49-56. doi: 10.1016/j.phymed.2018.12.002

Mustofa, A., Benoit-Vical, F., Pelissier, Y., Kone-Bamba, D., and Mallie, M. (2000). Antiplasmodial activity of plant extracts used-in west African traditional medicine. J. Ethnopharmacol. 73, 145-151. doi: 10.1016/S0378-8741(00) 00296-8

Nagai, A., Yokoyama, N., Matsuo, T., Bork, S., Hirata, H., Xuan, X., et al. (2003). Growth-inhibitory effects of artesunate, pyrimethamine, and pamaquine against Babesia equi and Babesia caballi in in vitro cultures. Antimicrob. Agents Chemother. 47, 800-803. doi: 10.1128/AAC.47.2.800-803.2003

Nehrbass-Stuedli, A., Boykin, D., Tidwell, R. R., and Brun, R. (2011). Novel diamidines with activity against Babesia divergens in vitro and Babesia microti in vivo. Antimicrob. Agents Chemother. 55, 3439-3445. doi: 10.1128/AAC.01482-10

Nishioka, T., Kawabata, J., and Aoyama, Y. (1998). Baicalein, an alpha-glucosidase inhibitor from Scutellaria baicalensis. J. Nat. Prod. 61, 1413-1415. doi: 10.1021/ np980163p

Olliaro, P. L., Haynes, R. K., Meunier, B., and Yuthavong, Y. (2001). Possible modes of action of the artemisinin-type compounds. Trends Parasitol. 17, 122126. doi: 10.1016/S1471-4922(00)01838-9

Osafo, N., Mensah, K. B., and Yeboah, O. K. (2017). Phytochemical and Pharmacological Review of Cryptolepis sanguinolenta (Lindl.) Schlechter. Adv. Pharmacol. Sci. 2017, 3026370. doi: 10.1155/2017/3026370

Pan, B., Shi, X., Ding, T., and Liu, L. (2019). Unraveling the action mechanism of Polygonum cuspidatum by a network pharmacology approach. Am. J. Transl. Res. 11, 6790-6811.

Pang, H., Xue, W., Shi, A., Li, M., Li, Y., Cao, G., et al. (2016). Multiple-AscendingDose Pharmacokinetics and Safety Evaluation of Baicalein Chewable Tablets in 
Healthy Chinese Volunteers. Clin. Drug Investig. 36, 713-724. doi: 10.1007/ s40261-016-0418-7

Parveen, N., and Bhanot, P. (2019). Babesia microti-Borrelia burgdorferi Coinfection. Pathogens 8 (3), 117. doi: 10.3390/pathogens8030117

Passos, C. L., Ferreira, C., Soares, D. C., and Saraiva, E. M. (2015). Leishmanicidal Effect of Synthetic trans-Resveratrol Analogs. PloS One 10, e0141778. doi: 10.1371/journal.pone.0141778

Price, R., van Vugt, M., Phaipun, L., Luxemburger, C., Simpson, J., McGready, R., et al. (1999). Adverse effects in patients with acute falciparum malaria treated with artemisinin derivatives. Am. J. Trop. Med. Hyg. 60, 547-555. doi: 10.4269/ ajtmh.1999.60.547

Puri, B. K., White, N., and Monro, J. A. (2019). The effect of supplementation with Scutellaria baicalensis on hepatic function. Med. Hypotheses 133, 109402. doi: 10.1016/j.mehy.2019.109402

Raffalli, J., and Wormser, G. P. (2016). Persistence of babesiosis for $>2$ years in a patient on rituximab for rheumatoid arthritis. Diagn. Microbiol. Infect. Dis. 85, 231-232. doi: 10.1016/j.diagmicrobio.2016.02.016

Rasoanaivo, P., Wright, C. W., Willcox, M. L., and Gilbert, B. (2011). Whole plant extracts versus single compounds for the treatment of malaria: synergy and positive interactions. Malar. J. 10 Suppl 1, S4. doi: 10.1186/1475-2875-10-S1-S4

Restani, P., Di Lorenzo, C., Garcia-Alvarez, A., Badea, M., Ceschi, A., Egan, B., et al. (2016). Adverse Effects of Plant Food Supplements Self-Reported by Consumers in the PlantLIBRA Survey Involving Six European Countries. PloS One 11, e0150089. doi: 10.1371/journal.pone.0150089

Rizk, M. A., El-Sayed, S. A., Terkawi, M. A., Youssef, M. A., El Said el Sel, S., Elsayed, G., et al. (2015). Optimization of a Fluorescence-Based Assay for Large-Scale Drug Screening against Babesia and Theileria Parasites. PloS One 10, e0125276. doi: 10.1371/journal.pone.0125276

Rizk, M. A., AbouLaila, M., El-Sayed, S. A. E., Guswanto, A., Yokoyama, N., and Igarashi, I. (2018). Inhibitory effects of fluoroquinolone antibiotics on Babesia divergens and Babesia microti, blood parasites of veterinary and zoonotic importance. Infect. Drug Resist. 11, 1605-1615. doi: 10.2147/IDR.S159519

Rizk, M. A., Ji, S. W., Liu, M. M., El-Sayed, S. A., Li, Y. C., Byamukama, B., et al. (2020). Closing the empty anti-Babesia gibsoni drug pipeline in vitro using fluorescence-based high throughput screening assay. Parasitol. Int. 75, 102054. doi: 10.1016/j.parint.2020.102054

Sanchez-Vicente, S., Tagliafierro, T., Coleman, J. L., Benach, J. L., and Tokarz, R. (2019). Polymicrobial Nature of Tick-Borne Diseases. mBio 10 (5), e02055-19. doi: $10.1128 / \mathrm{mBio} .02055-19$

Sawer, I. K., Berry, M. I., Brown, M. W., and Ford, J. L. (1995). The effect of cryptolepine on the morphology and survival of Escherichia coli, Candida albicans and Saccharomyces cerevisiae. J. Appl. Bacteriol. 79, 314-321. doi: 10.1111/j.1365-2672.1995.tb03143.x

Scott, J. D., and Scott, C. M. (2018). Human Babesiosis Caused by Babesia duncani Has Widespread Distribution across Canada. Healthcare-Basel 6 (2), 49. doi: 10.3390/healthcare6020049

Shan, B., Cai, Y. Z., Brooks, J. D., and Corke, H. (2008). Antibacterial properties of Polygonum cuspidatum roots and their major bioactive constituents. Food Chem. 109, 530-537. doi: 10.1016/j.foodchem.2007.12.064

Shin, N. R., Gu, N., Choi, H. S., and Kim, H. (2020). Combined effects of Scutellaria baicalensis with metformin on glucose tolerance of patients with type 2 diabetes via gut microbiota modulation. Am. J. Physiol. Endocrinol. Metab. 318, E52-E61. doi: 10.1152/ajpendo.00221.2019

Shoemaker, M., Hamilton, B., Dairkee, S. H., Cohen, I., and Campbell, M. J. (2005). In vitro anticancer activity of twelve Chinese medicinal herbs. Phytother. Res. 19, 649-651. doi: 10.1002/ptr.1702

Skinner, T. S., Manning, L. S., Johnston, W. A., and Davis, T. M. (1996). In vitro stage-specific sensitivity of Plasmodium falciparum to quinine and artemisinin drugs. Int. J. Parasitol. 26, 519-525. doi: 10.1016/0020-7519(96)89380-5

Smith, R. P., Hunfeld, K. P., and Krause, P. J. (2020). Management strategies for human babesiosis. Expert Rev. Anti Infect. Ther. 18, 625-636. doi: 10.1080/ 14787210.2020 .1752193

Smol'ianinov, E. S., Gol'dberg, V. E., Matiash, M. G., Ryzhakov, V. M., Boldyshev, D. A., Litvinenko, V. I., et al. (1997). [Effect of Scutellaria baicalensis extract on the immunologic status of patients with lung cancer receiving antineoplastic chemotherapy]. Eksp. Klin. Farmakol. 60, 49-51.

Stebbings, S., Beattie, E., McNamara, D., and Hunt, S. (2016). A pilot randomized, placebo-controlled clinical trial to investigate the efficacy and safety of an extract of Artemisia annua administered over 12 weeks, for managing pain, stiffness, and functional limitation associated with osteoarthritis of the hip and knee. Clin. Rheumatol. 35, 1829-1836. doi: 10.1007/s10067-015-3110-z

Swei, A., O'Connor, K. E., Couper, L. I., Thekkiniath, J., Conrad, P. A., Padgett, K. A., et al. (2019). Evidence for transmission of the zoonotic apicomplexan parasite Babesia duncani by the tick Dermacentor albipictus. Int. J. Parasitol. 49, 95-103. doi: 10.1016/j.ijpara.2018.07.002

Tagboto, S., and Townson, S. (2001). Antiparasitic properties of medicinal plants and other naturally occurring products. Adv. Parasitol. 50, 199-295. doi: 10.1016/S0065-308X(01)50032-9

Tempesta, M. S. (2010). The clinical efficacy of Cryptolepis sanguinolenta in the treatment of malaria. Ghana Med. J. 44, 1-2.

Tona, L., Kambu, K., Ngimbi, N., Cimanga, K., and Vlietinck, A. J. (1998). Antiamoebic and phytochemical screening of some Congolese medicinal plants. J. Ethnopharmacol. 61, 57-65. doi: 10.1016/S0378-8741(98)00015-4

Trinh, H., Yoo, Y., Won, K. H., Ngo, H. T. T., Yang, J. E., Cho, J. G., et al. (2018). Evaluation of in-vitro antimicrobial activity of Artemisia apiacea $\mathrm{H}$. and Scutellaria baicalensis G. extracts. J. Med. Microbiol. 67, 489-495. doi: 10.1099/ jmm.0.000709

Vannier, E., and Krause, P. J. (2009). Update on babesiosis. Interdiscip. Perspect. Infect. Dis. 2009, 984568. doi: 10.1155/2009/984568

Vannier, E., and Krause, P. J. (2012). Human babesiosis. N. Engl. J. Med. 366, 2397-2407. doi: 10.1056/NEJMra1202018

Vannier, E. G., Diuk-Wasser, M. A., Ben Mamoun, C., and Krause, P. J. (2015). Babesiosis. Infect. Dis. Clin. North Am. 29, 357-370. doi: 10.1016/ j.idc. 2015.02 .008

Virji, A. Z., Thekkiniath, J., Ma, W. X., Lawres, L., Knight, J., Swei, A., et al. (2019). Insights into the evolution and drug susceptibility of Babesia duncani from the sequence of its mitochondrial and apicoplast genomes. Int. J. Parasitol. 49, 105-113. doi: 10.1016/j.jpara.2018.05.008

W.H.O.W.P.S (2012). Effectiveness of Non-Pharmaceutical Forms of Artemisia annua L. against malaria. Available at: https://www.who.int/malaria/position statement_herbal_remedy_artemisia_annua_l.pdf (Accessed August 28, 2020).

Walle, T. (2011). Bioavailability of resveratrol. Ann. N. Y. Acad. Sci. 1215, 9-15. doi: $10.1111 / j .1749-6632.2010 .05842 . x$

Wang, J., Zhang, C. J., Chia, W. N., Loh, C. C., Li, Z., Lee, Y. M., et al. (2015). Haem-activated promiscuous targeting of artemisinin in Plasmodium falciparum. Nat. Commun. 6, 10111. doi: 10.1038/ncomms10111

World Health Organization (WHO) (2015). Guidelines for the Treatment of Malaria 2015 (Geneva, Switzerland: World Health Organization).

Wright, C. W., Phillipson, J. D., Awe, S. O., Kirby, G. C., Warhurst, D. C., QuetinLeclercq, J., et al. (1996). Antimalarial activity of cryptolepine and some other anhydronium bases. Phytother. Res. 10, 361-363. doi: 10.1002/(SICI) 1099-1573(199606)10:4<361::AID-PTR845>3.0.CO;2-N

Wu, J., Hu, D., and Wang, K. X. (2008). [Study of Scutellaria baicalensis and Baicalin against antimicrobial susceptibility of Helicobacter pylori strains in vitro]. Zhong Yao Cai 31, 707-710.

Yang, M., Guo, M. Y., Luo, Y., Yun, M. D., Yan, J., Liu, T., et al. (2017). Effect of Artemisia annua extract on treating active rheumatoid arthritis: A randomized controlled trial. Chin. J. Integr. Med. 23, 496-503. doi: 10.1007/s11655-0162650-7

Yao, J. M., Zhang, H. B., Liu, C. S., Tao, Y., and Yin, M. (2015). Inhibitory effects of 19 antiprotozoal drugs and antibiotics on Babesia microti infection in BALB/c mice. J. Infect. Dev. Ctries 9, 1004-1010. doi: 10.3855/jidc.5500

Zhang, Y., Bai, C., Shi, W., Alvarez-Manzo, H., and Zhang, Y. (2020). Identification of Essential Oils Including Garlic Oil and Black Pepper Oil with High Activity against Babesia duncani. Pathogens 9 (6), 466. doi: 10.3390/ pathogens 9060466

Zhao, Q., Chen, X. Y., and Martin, C. (2016). Scutellaria baicalensis, the golden herb from the garden of Chinese medicinal plants. Sci. Bull. (Beijing) 61 (18), 1391-1398. doi: 10.1007/s11434-016-1136-5

Zhao, Q., Luan, X., Zheng, M., Tian, X. H., Zhao, J., Zhang, W. D., et al. (2020), Synergistic Mechanisms of Constituents in Herbal Extracts during Intestinal Absorption: Focus on Natural Occurring Nanoparticles. Pharmaceutics 12 (2), 128. doi: 10.3390/pharmaceutics 12020128

Conflict of Interest: JL is the owner of two naturopathic medical practices, FOCUS Health Group and Door One Concierge, which provides treatment to 
patients with tick-borne diseases. JL does receive profits from medical services and botanical preparations he exclusively makes available to patients in these two practices and does not currently sell botanical products commercially.

The remaining authors declare that the research was conducted in the absence of any commercial or financial relationships that could be construed as a potential conflict of interest.
Copyright () 2021 Zhang, Alvarez-Manzo, Leone, Schweig and Zhang. This is an open-access article distributed under the terms of the Creative Commons Attribution License (CC BY). The use, distribution or reproduction in other forums is permitted, provided the original author(s) and the copyright owner(s) are credited and that the original publication in this journal is cited, in accordance with accepted academic practice. No use, distribution or reproduction is permitted which does not comply with these terms. 